\title{
General Description of the Complex Faulting Process and Some Empirical Relations in Seismology
}

\author{
Junji Koyama \\ Geophysical Institute, Faculty of Science, Tohoku University, \\ Aoba-ku, Sendai 980, Japan
}

\begin{abstract}
A theory of the complex faulting process of large earthquakes has been developed. The faulting process is characterized by a deterministic source of coherent rupture on a fault plane as well as by a stochastic source of incoherent ruptures in fault heterogeneous areas. The theory provides an earthquake source spectrum of the complex faulting process in a general manner: Theoretical representations of strong-motion acceleration, total power of acceleration, seismic energy, seismic-wave energy, surface-wave magnitude and body-wave magnitude are derived in terms of deterministic and stochastic source parameters. A low-frequency approximation of the source spectrum provides the description of the deterministic source by parameters of seismic moment, fault length, fault width, fault dislocation, stress drop, dislocation velocity, and rupture propagation velocity. Meanwhile, a high-frequency approximation provides the description of the stochastic source by patch corner frequency, cutoff frequency, variance of stress drop, and variance of dislocation velocity.

The present theory of the complex faulting process is constrained empirically against observations. It is shown that empirical relations between seismic moment, fault dimension, seismic energy, and earthquake magnitude are the manifestation of similarity rules among source parameters of the complex faulting process: The geometrical similarity is for the fault shape, the kinematical similarity is for the dislocation and rupture velocities, the dynamical similarity is for the average stress drop, and a new similarity rule, stochastic similarity is derived for the stochastic source parameters. The complex faulting process thus far constrained fully explains the general properties of large earthquakes along subduction zones.
\end{abstract}

\section{Introduction}

The excitation and propagation of high-frequency seismic waves are of special interest in recent earthquake seismology. In particular, the description and simulation of strong ground motion are very important not only for problems directly relevant to earthquake engineering, but also to fracture mechanics of earthquake faultings (Aki, 1989; Scholz, 1990). Understanding of the earthquake sources has been developed by investigating the complexity of faulting processes for the case of large earthquakes (e.g., Boatwright, 1988; Das and Aki, 1977; Fukao and Furumoto, 1985; Kikuchi

Received September 28, 1992; Accepted May 9, 1994 
and Kanamori, 1986; Koyama, 1985; Mikumo and Miyatake, 1987; Takeo, 1987). Laboratory results on rock failures have also advanced the understanding of faulting mechanisms (e.g., Ohnaka et al., 1987; Tse and Rice, 1986). Various attempts have been made to simulate, theoretically and empirically, the propagation of short-period seismic waves in the heterogeneous real earth (e.g., Chapman, 1985; Kawase and Aki, 1990; Sato, 1990; Spudich and Frazer, 1984).

These studies revealed that the excitation of short waves are strongly dependent on the complex faulting process due to small-scale fault heterogeneities and that such waves suffer from strong attenuation and scattering effects along the propagation path. This is the dilemma we face, and it leads us to admit that the source and propagation effects are not completely distinguishable from actual observations. Therefore, it is not yet satisfactory to describe the real earthquake source in terms of heterogeneous stresses, material properties, and non-uniform rupture propagations on the fault plane.

In this study, we shall investigate a complex faulting process of large earthquakes considering a deterministic source (coherent rupture) and a stochastic source (incoherent rupture) occurring simultaneously on the same fault plane. This is to describe the complex faulting process by physical parameters, which are independent of propagation effects. Detailed studies on propagation effects are provided in the references. Therefore, we focus the discussion only on the complex faulting process of large earthquakes. Then, the parameters of the present theory are constrained from observations in a general manner.

The present theory is not the only description of heterogeneous earthquake sources. Papageorgiou and Aki (1983), for example, considered a specific size of fault heterogeneities, where a local stress drop is defined. Their theory may be applied to some particular earthquakes with a periodic distribution of fault heterogeneities, however, they did not study the size variation of fault heterogeneities nor the fluctuation of local stress drops on the fault. Numerical studies on fault heterogeneities have also been conducted (e.g., Andrews, 1981; Boatwright, 1988; Suzuki and Hirasawa, 1984). These studies have developed the understanding of fault heterogeneities on short-period seismic excitation, however, they have not extended the knowledge on the scaling relation of stochastic source parameters.

This study is intended to extend our understanding on the complex faulting process of large earthquakes. The theory provides the description of seismic radiations, accelerations, seismic energy, and earthquake magnitude in a general manner. Although it seems to be largely expository, this study starts with a review of the general description of the complex faulting process to clarify the deterministic and stochastic parts of the faulting processes. We then discuss the general scaling law of earthquake source parameters.

\section{Earthquake Source Spectrum of Complex Faulting Process}

\subsection{Source spectrum of heterogeneous faulting}

An earthquake fault is defined by a dislocation distribution $D(\xi, t)$ at a point $\xi$ on the plane and at a time $t$. Suppose that the fault plane extends for a length $L$ and a width $W$ within an infinite homogeneous medium. For simplicity, the faulting rupture 


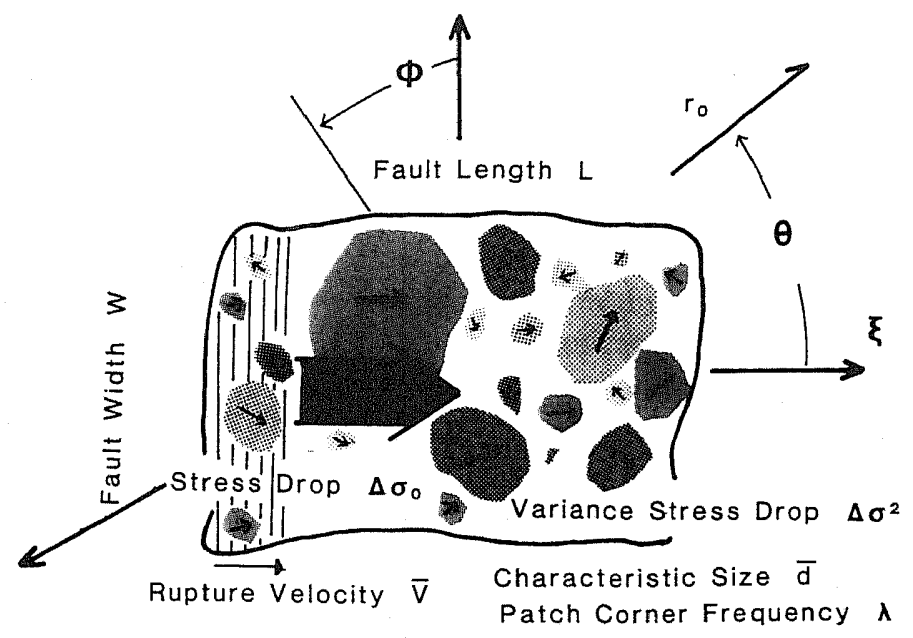

Fig. 1. Schematic illustration of the heterogeneous earthquake fault. Fault length and width, stress drop, and average dislocation are macroscopic source parameters of the complex faulting process. Random fault patches represent small-scale fault heterogeneities. Patch corner frequency and yariance stress drop are stochastic source parameters of the complex faulting process.

is assumed to propagate unilaterally along the $\xi$-axis for a distance $L$. Figure 1 illustrates a heterogeneous faulting where a smooth-and-coherent rupture extends over a rectangular fault plane. In addition, non-uniform and incoherent ruptures propagate over localized small areas on the fault.

The displacement component of P-waves in the far-field from this kind of a shear fault is generally written as (Aki and Richard, 1980)

$$
u_{r}=\frac{(\beta / \alpha)^{3}}{4 \pi \beta r_{0}} \sin 2 \theta \sin \phi I_{\alpha}(t)
$$

and those of S-waves as

$$
\begin{gathered}
u_{\theta}=\frac{1}{4 \pi \beta r_{0}} \cos 2 \theta \sin \phi I_{\beta}(t), \\
u_{\phi}=\frac{1}{4 \pi \beta r_{0}} \cos \theta \cos \phi I_{\beta}(t)
\end{gathered}
$$

and

$$
I_{c}(t)=W \int_{0}^{L} \dot{D}\left(\xi, t-\frac{r-\xi \cos \theta}{c}\right) \mathrm{d} \xi
$$

where $\dot{D}(\xi, t)$ is a dislocation velocity averaged along the fault width on the fault plane (Aki, 1967). In the above equations, the distance of an observer is $r_{0}$ from the center 


\section{Coherent}
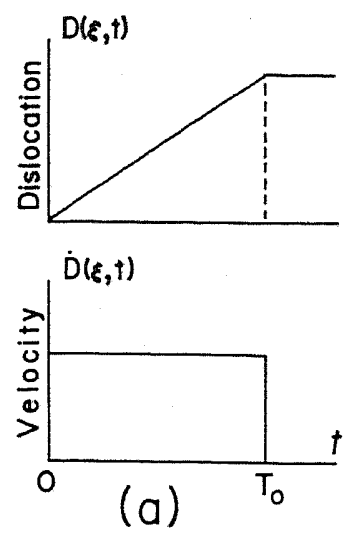

Coherent / incoherent
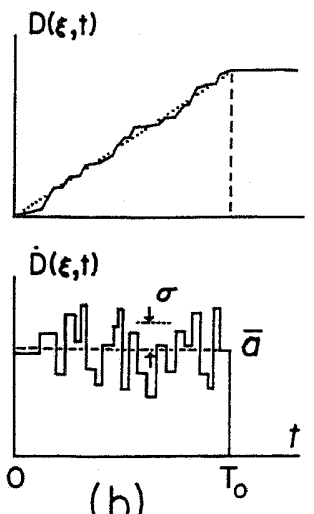

Fig. 2. Dislocation time-function and dislocation velocity time-function at a point on a fault. Average dislocation velocity $\bar{a}$ and variance dislocation velocity $\sigma^{2}$ represent the deterministic and the stochastic parts of the complex faulting process. The rise time of dislocation on a fault is $T_{0}$ and $\bar{v}$ is an average rupture velocity.

of the fault, $\alpha$ and $\beta$ indicate P- and S-wave velocities, where $c$ stands for either of them. Polar coordinates $(r, \theta, \phi)$ are introduced in Fig. 1: $\theta$ is the polar angle of an observer measured from the fault strike, and $\phi$ is the azimuth angle. Although we should consider more strictly the dislocation velocity function and the rupture propagation effect along the fault width, Eq. (2.3) suffices as an expression for a very long fault without losing generality.

It would be unlikely that the rupture of small-scale localized areas is coherent over the full length of the fault. We designate such a small-scale heterogeneous area as a fault patch. It would be more probable that fault patches are localized and correlated only over limited segments of the fault (Koyama, 1985). Figure 2 shows schematics of dislocation functions for a coherent rupture and for incoherent ruptures superimposed on a coherent rupture.

Since an earthquake is essentially a transient phenomenon, the dislocation function $D(\xi, t)$ in Fig. 2 should be zero when $t<0$. Also it will be a constant value $D(\xi)$ for $t>T_{0}$, where $T_{0}$ is a time necessary to complete the dislocation on the fault. The parameter $T_{0}$ is related to a characteristic rupture time of the fault width as

$$
T_{0}=q W / \bar{v},
$$

where $\bar{v}$ is an average rupture velocity and $q$ is a constant. In the latter section, $q$ is assumed to be $1 / 2$ (Savage, 1972).

If the faulting process is a coherent rupture, $\dot{D}(\xi, t)$ would be represented as a dislocation velocity of magnitude $\bar{a}$, as in Fig. 2(a). The coherent rupture at $\xi$ radiates a rectangular pulse with an amplitude of $\bar{a}$ and with a time duration $T_{0}$ (Haskell, 1964; Hirasawa and Stauder, 1965). On the other hand, if the faulting is highly irregular, $\dot{D}$ 
would be described by the coherent part and incoherent part as in Fig. 2(b). The coherent rupture is the same as that in Fig. 2(a), characterized by $\bar{a}$. Meanwhile, the rupture of localized fault patches radiates a rectangular pulse superimposed on $\bar{a}$ representing the fluctuation of dislocation velocities. Boore and Joyner (1978) and Koyama (1985) derived the dislocation velocity function in this case by the sum of the smooth rectangular pulse (coherent rupture) and random rectangular pulses (incoherent ruptures). We consider that the amplitude of random pulses in Fig. 2(b) is characterized by a variance of dislocation velocities $\sigma^{2}$. We also assume that pulse widths relate to the rupture duration on the patchy areas. Statistical theory of communications (Bendat, 1958) has been extended to obtain an autocovariance function of the random-pulse time series in Fig. 2(b), and is expressed as

$$
C(\tau)= \begin{cases}\left(T_{0}-|\tau|\right)\left\{\bar{a}^{2}+\sigma^{2} \exp (-\lambda|\tau|)\right\}, & 0 \leq|\tau| \leq T_{0} \\ 0, & T_{0}<|\tau|,\end{cases}
$$

where $\lambda$ is a mean number of random-pulse occurrences in a unit of time. This is expected to be

$$
\lambda=\bar{v} / \bar{d}
$$

where $\bar{d}$ is a characteristic length of fault patches. This equation is understood to be the inverse of a mean rupture time of fault patches. $C(\tau)$ represents an autocovariance of the smooth rupture when $\sigma^{2} \rightarrow 0$.

The autocovariance in Eq. (2.5) is for a limited fault segment at $\xi$. If we consider that the heterogeneous faulting process is homogeneous in the time domain and the space domain, the contribution of incoherent ruptures can be expressed similarly as Eq. (2.5) along the fault length. The autocovariance for the full-length of the fault is expressed by summing up Eq. (2.5) along the fault length $L$ where the faulting rupture propagates at a velocity $\bar{v}$. The source spectrum $\left|A_{c}(\omega)\right|$ of the complex faulting process can be expressed by the Fourier transform of the autocovariance function as

$$
\begin{aligned}
&\left|A_{c}(\omega)\right|^{2}= W^{2} \mid \int_{-\infty}^{\infty} \mathrm{d} \tau \exp (-i \omega \tau) \\
& \times \int_{-L}^{L} \mathrm{~d} \xi \exp \left(i k_{c} \xi\right)(L-|\xi|)\left(T_{0}-|\tau-\xi / \bar{v}|\right)\left\{\vec{a}^{2}+\sigma^{2} \exp (-\lambda|\tau-\xi / \bar{v}|)\right\} \mid \\
& \text { for }|\tau-\xi / \bar{v}| \leq T_{0}
\end{aligned}
$$

where $k_{c}$ is a wave number of $\omega \cos \theta / c$, and $\omega$ is the angular frequency. When the fault width is much larger than the characteristic patch size, $W_{\gg} \bar{d}$, the above equation is rewritten as (Koyama, 1985)

$$
\begin{aligned}
\left|A_{c}(\omega)\right|^{2}= & \left(L W T_{0} \bar{a}\right)^{2} \frac{\sin ^{2}\left\{\left(k_{c}-\omega / \bar{v}\right) L / 2\right\}}{\left\{\left(k_{c}-\omega / \bar{v}\right) L / 2\right\}^{2}} \\
& \times\left[\frac{\sin ^{2}\left(\omega T_{0} / 2\right)}{\left(\omega T_{0} / 2\right)^{2}}+\frac{2 \sigma^{2}}{T_{0}^{2} \bar{a}^{2}}\left\{\frac{\omega^{2}-\lambda^{2}}{\left(\omega^{2}+\lambda^{2}\right)^{2}}+\frac{\lambda T_{0}}{\omega^{2}+\lambda^{2}}\right\}\right] .
\end{aligned}
$$


It should be noted that all frequencies throughout this study are the angular frequency except for some special cases expressed by frequency $f$.

The displacement spectrum $\Omega$ of P- and S-waves radiated from this complex faulting process can be expressed by applying Eqs. (2.8) to (2.1) and Eq. (2.2) as

$$
\begin{gathered}
\Omega(P, S)=\frac{M_{o}}{4 \pi \rho r_{0}\left(\alpha^{3}, \beta^{3}\right)}\left|R_{\theta \phi}(P, S)\right|\left|B_{c}(\omega)\right| \\
\left|B_{c}(\omega)\right|=\frac{\left|\sin \left\{\left(k_{c}-\omega / \bar{v}\right) L / 2\right\}\right|}{\left|\left(k_{c}-\omega / \bar{v}\right) L / 2\right|}\left[\frac{\sin ^{2}\left(\omega T_{0} / 2\right)}{\left(\omega T_{0} / 2\right)^{2}}+\frac{2 \sigma^{2}}{T_{0}^{2} \bar{a}^{2}}\left\{\frac{\omega^{2}-\lambda^{2}}{\left(\omega^{2}+\lambda^{2}\right)^{2}}+\frac{\lambda T_{0}}{\omega^{2}+\lambda^{2}}\right\}\right]^{1 / 2}
\end{gathered}
$$

where $M_{o}$ is a seismic moment, and $R_{\theta \phi}(P, S)$ is the radiation pattern coefficient either for $\mathrm{P}$ - or S-waves from a point double-couple.

The excitation of seismic body-waves could generally be represented by a seismic moment tensor (Aki and Richard, 1980; Dziewonski et al., 1981; Kikuchi and Kanamori, 1991; Koyama, 1987; Sipkin, 1982). The moment tensor appropriate for a general shear-fault is the combination of two point double-couples orthogonal to each other (Randoll and Knopoff, 1970). This understanding enables us to formulate the heterogeneous faulting process by combining the contribution of displacement components from the other (minor) double-couple to Eq. (2.9). In order to make the discussion simple and suitable for actual applications, we no longer investigate the moment tensor representation of heterogeneous faultings.

\subsection{Source parameters of heterogeneous faulting}

The source spectrum in Eq. (2.8) has a characteristic corner frequency of

$$
\omega_{c}=2 \bar{v} /\{L(1-\bar{v} \cos \theta / c)\},
$$

where the spectral envelope changes its general trend. A source spectrum of the coherent rupture is obtained, when the variance dislocation velocity $\sigma^{2}$ is zero. Fault length, fault width, average stress drop, average dislocation velocity, and rupture velocity are the parameters which describe the deterministic part of the complex faulting process.

Since $T_{0}$ is a time duration of dislocation motion, $T_{0} \bar{a}$ describes an average amount of dislocation on the fault. Therefore, a quantity $T_{0} \bar{a} / W$ would be a measure of an average strain release on the fault. Multiplying rigidity $\mu$ by the strain, a relationship between the average stress drop and the strain release is obtained (Kanamori and Anderson, 1975) as

$$
\Delta \sigma_{0}=\mu\left(T_{0} \bar{a}\right) / W .
$$

The stress drop in Eq. (2.12) is nothing but a formal representation for the present fault model. When the stress drop of real earthquakes is considered, it is necessary to apply the scale factor resulting from the fault shape to the expression in Eq. (2.12). The above equation could also be obtained from Eqs. (2.8) and (2.9) with the definition of seismic moment

$$
M_{o}=\Delta \sigma_{0} W^{2} L\left(=\mu L W T_{0} \bar{a}\right)
$$


We take the dislocation model to describe the earthquake source in this study. A crack model is also justified describing the earthquake source. Mathematical theory of dislocations states the equivalence of these two approaches for the coherent rupture (Maruyama, 1963). Considering the complex faulting process, there is no theory that. guarantees the validity of the equivalency in a general manner. However, the formal representation in Eq. (2.5) specifies the dislocation velocity on the heterogeneous fault, and describes the kinematics of the complex faulting process. At the same time, the average stress drop, defined in Eq. (2.12), specifies the dynamical condition on the fault plane as an average.

Similar to the variance dislocation velocity $\sigma^{2}$ in Eq. (2.5), the variance stress drop $\left\langle\Delta \sigma^{2}\right\rangle$ can be formally defined from the source spectrum in Eq. (2.8) to characterize the stochastic part of the complex faulting process (Koyama and Izutani, 1990):

$$
\left\langle\Delta \sigma^{2}\right\rangle=2 \mu^{2} \sigma^{2} T_{0} /\left(W^{2} \lambda\right) \text {. }
$$

Because the variance stress drop is characterized by a random force with zero mean centered at $\Delta \sigma_{0}$, the fluctuation on localized fault patches would not contribute to the seismic moment. This understanding gives an inequality relationship of

$$
\Delta \sigma^{2} \gg\left\langle\Delta \sigma^{2}\right\rangle \text {. }
$$

The source spectrum in Eq. (2.8) shows a frequency-dependence of $\omega^{\circ}-$ $\omega^{-2}-\omega^{-\delta}-\omega^{-2}$ with increasing frequency $\omega$. The power coefficient $\delta$ is measured to be about 1 . There appear two characteristic corner frequencies; the first corner frequency results from the rupture duration of the whole faulting defined in Eq. (2.11), and the second corner frequency from the average rupture time of random fault patches. The latter corner frequency is strictly related to $\lambda$ and is essential in characterizing the complex faulting process.

The definition of $\lambda$ in Eq. (2.6) is for the patch corner frequency (Koyama, 1985) introduced to the seismological literature in order to describe the scale effect of fault heterogeneities. The complex faulting process is thus formulated by the microscopic parameters of variance dislocation velocity $\sigma^{2}$, variance stress drop $\left\langle\Delta \sigma^{2}\right\rangle$, and patch corner frequency $\lambda$ for the stochastic part of the complex faulting process, in addition to the macroscopic parameters for the deterministic part.

\subsection{Scaling law of earthquake source parameters}

\subsubsection{Geometrical similarity}

An empirical relation between seismic moment $M_{o}$ and source dimension $S$ has been obtained by Abe (1975):

$$
\log M_{o}=(3 / 2) \log S+22.1,
$$

where the units of $M_{o}$ and $S$ are dyne $\cdot \mathrm{cm}$ and $\mathrm{km}^{2}$, respectively. Because $S$ is reasonably understood to be a measure of $L W$, this relation suggests that the seismic moment is a function of only $L$ and $W$. Taking a logarithm of the seismic moment in Eq. (2.13), the above equation gives

$$
W / L=p \text { (constant) }
$$


This is a geometrical similarity of faultings irrespective of the size of earthquake sources.

\subsubsection{Dynamical similarity}

The geometrical similarity suggests that the seismic moment $M_{o}$ is proportional to $W^{3}$ and that the coefficient for the proportionality is the stress drop $\Delta \sigma_{0}$ from Eq. (2.13). This is a dynamical similarity of the constant stress drop as

$$
\Delta \sigma_{0}=\text { constant }
$$

\subsubsection{Kinematical similarity}

After the rise time $T_{0}$ in Eq. (2.4) is substituted into the expression for the stress drop in Eq. (2.12), the above dynamical similarity is equivalent to a kinematical similarity for the dislocation velocity

$$
\bar{a}=\text { constant . }
$$

Another kinematical similarity has been independently obtained from the seismic directivity effect of large earthquakes

$$
\bar{v}=\text { constant . }
$$

It has been pointed out that rupture velocity, stress drop, and dislocation velocity are stable parameters, not only for earthquake faultings but also for rock failures in experiments (Ohnaka et al., 1987).

\subsubsection{Stochastic similarity}

The similarity rules so far discussed are for the macroscopic parameters of the complex faulting process. Scaling stochastic source parameters is a very difficult problem and still awaits the analysis of short-period seismic waves. Systematic estimate of a high-frequency source spectrum $M_{1}$ at about $1 \mathrm{~Hz}\left(\omega_{b}=2 \pi\right)$ has been made for large earthquakes which have occurred along subduction zones throughout the world (Koyama and Zheng, 1985). An empirical relation reproduced in Fig. 3 has been obtained in a wide range of seismic-moment values:

$$
M_{o} \propto M_{1}^{2} \text {. }
$$

This relation is the only restriction at present to investigate the scaling relation of large earthquakes in the short-period range. To constrain the seismic excitation in a high-frequency range, two models are considered for the patch corner frequency. In the first case, $\lambda$ is not a constant but relates to the fault dimension as

$$
\lambda=C_{p} \omega_{c},
$$

where $\omega_{c}$ is a corner frequency of an earthquake and $C_{p}$ is a constant, about 7 to 10 (Koyama, 1985). This is a scaling patch corner-frequency model (scaling P-model). Then the characteristic frequency $\omega_{b}(=2 \pi)$ for $M_{1}$ is generally much larger than $\lambda$ of large and great earthquakes. A high-frequency approximation of Eq. (2.8) is obtained at this frequency of $\omega_{b}$, where $\omega_{b} \gg 2 / T_{0}$ and $\omega_{b} \gg 2 \bar{v} / L$,

$$
S_{0} \simeq \frac{2 \bar{v}}{\omega_{b}}\left(\frac{2 \lambda \sigma^{2}}{\omega_{b}^{2}+\lambda^{2}}\right)^{1 / 2} W T_{0}^{1 / 2}
$$




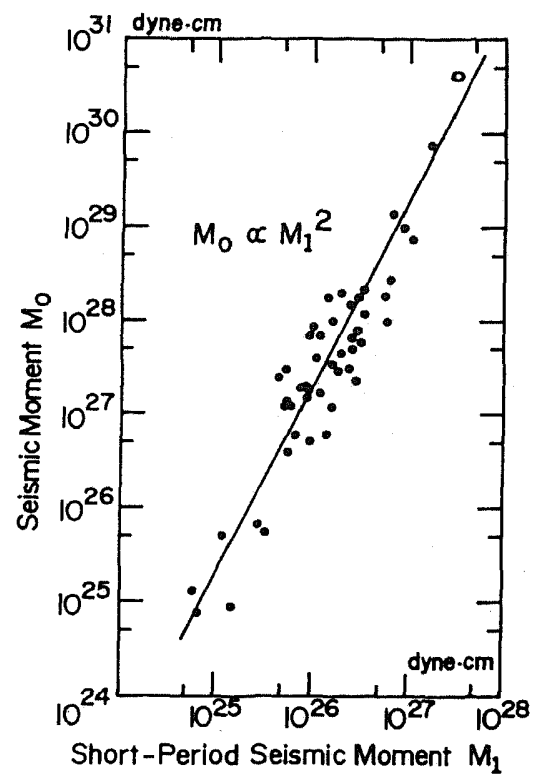

Fig. 3. Seismic moment and estimate of short-period seismic moment at $1 \mathrm{~Hz}$ (Koyama and Zheng, 1985). Open circle is the data for the 1960 Chilean earthquake.

Then, the source representation for the scaling P-model would be reduced to

$$
M_{1} \propto S_{0} \simeq \frac{2 \bar{v}}{\omega_{b}{ }^{2}}\left(2 \lambda \sigma^{2}\right)^{1 / 2} W T_{0}{ }^{1 / 2} .
$$

Since we have studied $M_{0} \propto W^{2} T_{0}$ from the deterministic similarity rules, the $M_{0}-M_{1}$ relation in Eq. (2.21) suggests an invariant parameter irrespective of the size of earthquake sources

$$
\lambda \sigma^{2}=\text { constant } .
$$

This is another similarity rule specially for the stochastic faulting parameters. This is the stochastic similarity of the complex faulting process.

In the other case, $\lambda$ is a constant for large and great earthquakes (constant P-model). In this case, $\lambda$ would be more or less $2 \pi$, for reasons we will discuss later. Assuming $\omega_{b}{ }^{2}+\lambda^{2}=2 \omega_{b}{ }^{2}$, since $\omega_{b}=2 \pi$, the high-frequency source spectrum in Eq. (2.23) is approximated as

$$
S_{0} \simeq \frac{2 \bar{v}}{\omega_{b}{ }^{2}}\left(\lambda \sigma^{2}\right)^{1 / 2} W T_{0}{ }^{1 / 2} .
$$

The same similarity rule as in Eq. (2.25) can be derived for the stochastic source parameters in this case of the constant P-model. This stochastic similarity is the clue to constrain the heterogeneous property of the complex faulting process. The value of 
the stochastic similarity will be determined in Sec. 6 from the observation.

\section{Acceleration Spectrum of Complex Faulting Process}

\subsection{Acceleration spectrum of heterogeneous faulting}

The acceleration spectrum of the complex faulting process can be obtained from the source spectrum in Eq. (2.8), differentiating twice by time (Koyama and Izutani, 1990)

$$
G_{h}(P, S)=\frac{M_{o}}{4 \pi \rho r_{0}\left(\alpha^{3}, \beta^{3}\right)}\left|R_{\theta \phi}(P, S)\right| \omega^{2}\left|B_{c}(\omega)\right|
$$

Due to the terms of $\omega^{2}\left|B_{c}(\omega)\right|$, the acceleration spectrum shows a frequencydependence of $\omega^{2}-\omega^{0}-\omega^{2-\delta}-\omega^{0}$ with increasing frequency. The corner frequency characterizes the spectral envelope between $\omega^{2}$ and $\omega^{0}$, while the patch corner frequency between $\omega^{2-\delta}$ and $\omega^{0}$. Figure 4 shows examples of acceleration spectra from the complex faulting process. Hereafter, the acceleration spectrum in Eq. (3.1) is designated as that of the stochastic faulting model.

Constant acceleration spectra have usually been found from strong motion records (e.g., Toro and McGuire, 1987), which corresponds to the spectral component $\omega^{0}$ beyond the patch corner frequency in the above theoretical spectrum of Eq. (3.1). In the intermediate frequency range from the corner to the patch corner frequencies, we observe an increase of spectral components in proportion to $\omega^{2-\delta}$ where $\delta$ is about 1 . This spectral behavior has been pointed out from acceleration records by Gusev (1983), Izutani (1984), and Papageorgiou and Aki (1985) and also from the mutual relations among different earthquake magnitudes by Aki (1972), Boore (1986), and Koyama et al. (1982).

Since strong motion acceleration is dominant in short-period S-waves, let us study the high-frequency approximation of S-wave acceleration in Eq. (3.1). Farfield approximation in Eq. (2.9) and high-frequency approximation here are always valid for short waves, because the effective rupture radiating short waves is not the whole fault but rather small-scale fault patches.

Strong ground motion of large earthquakes always shows complicated waveforms. Apparently wave trains are composed of random-phase short-waves arriving at every moment at an observer (Koyama and Zheng, 1985). This is mainly due to the generation of short waves by random rupturing fault patches. In addition, the focal mechanism of fault patches would not be the same as that of the coherent rupture, but would fluctuate in a random fashion. Such waves, after suffering from refraction, reflection and scattering, leave the source region. They would consequently show a weak amplitudedependence on the point-source focal mechanism of the deterministic source process. The total power of short-period acceleration is derived as an average:

$$
\begin{aligned}
P_{a}\left(r_{0}\right) & =\frac{1}{4 \pi} \int_{0}^{2 \pi} \mathrm{d} \phi \int_{0}^{\pi}\left\{P_{t}\left(u_{\theta}\right)+P_{t}\left(u_{\phi}\right)\right\} \sin \theta \mathrm{d} \theta \\
& \simeq g(\chi)\left(\frac{M_{o}}{4 \pi \rho r_{0} \beta^{3}}\right)^{2} \frac{\left\langle\Delta \sigma^{2}\right\rangle}{\Delta \sigma_{0}{ }^{2}} \frac{\lambda^{2}\left(\omega_{m}-\pi \lambda / 2\right) \bar{v}^{2}}{L^{2}},
\end{aligned}
$$




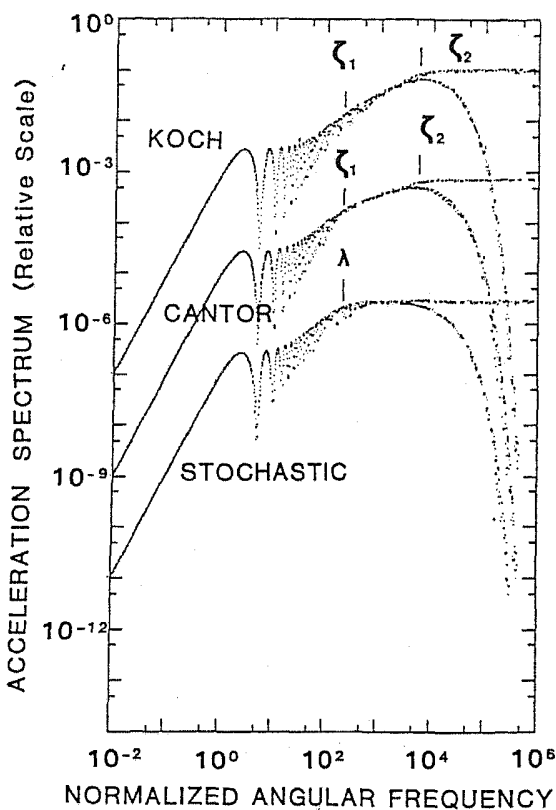

(a)

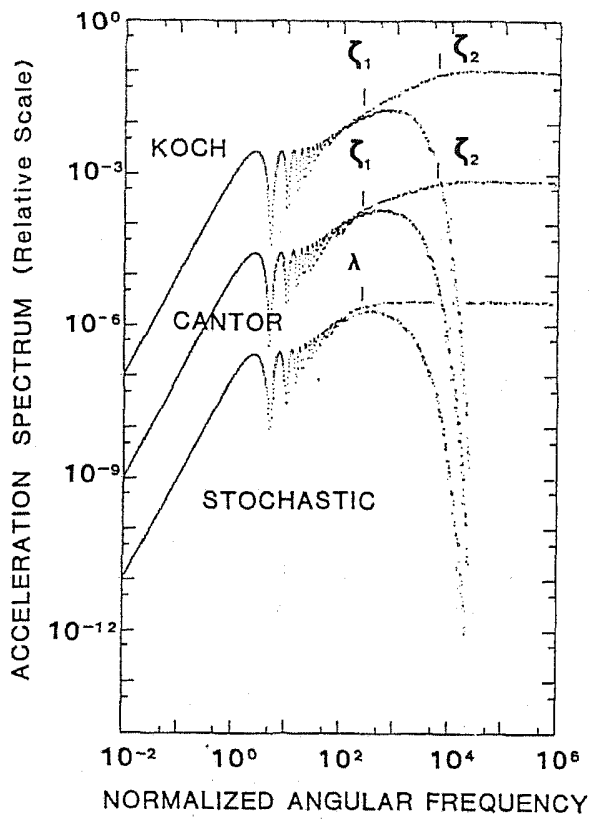

(b)

Fig. 4. Acceleration spectrum from the stochastic faulting model, and from the self-similar fault patch models characterized by the Cantor and Koch models. The stochastic faulting model shows the frequency-dependence of $\omega^{2}-\omega^{0}-$ $\omega^{2-\delta}-\omega^{0}$. The angular frequency here is normalized by $W / \beta$. The self-similar fault patch models show a frequency-dependence of $\omega^{2}-\omega^{0}-\omega^{2-\delta}-\omega^{\kappa / 2}-$ $\omega^{0}$, where $\kappa$ is the fractal dimension of self-similar fault patches. Characteristic frequencies of $\zeta_{1}$ and $\zeta_{2}$ are due to the largest and smallest fault patches. In high frequency range, all the spectra show a constant spectral density. Spectral rolling-down in high frequency range is due to slight attenuation in (a) and to large attenuation in (b). An empirical attenuation in the form of $\exp \left(-\omega t^{*}\right)$ is assumed, where $t^{*}=0.000039$ normalized by $W / \beta$ in (a) and $t^{*}=0.00069$ in (b), respectively.

$$
g(\chi)=\frac{96-100 \chi^{2}+16 \chi^{4}}{3 \pi \chi^{4}\left(1-\chi^{2}\right)}+\frac{8-3 \chi^{2}}{\pi \chi^{5}} \ln \left(\frac{1-\chi}{1+\chi}\right)^{2},
$$

and $\chi$ is a velocity ratio of $\bar{v} / \beta$ and

$$
P_{t}=\frac{1}{2 \pi} \int_{-\omega_{m}}^{\omega_{m}} G_{h}(S)^{2} \mathrm{~d} \omega,
$$

where $\omega_{m}$ is an empirical cutoff frequency (Hanks, 1982) beyond which the spectral component does not contribute effectively to the actual strong ground motion.

Let us consider an attenuation of high-frequency acceleration. An exponential

Vol. 42, No. 2, 1994 
decaying behavior of a type $\exp \left(-\omega t^{*} / 2\right)$ is temporally assumed, where $t^{*}$ is a travel time of seismic waves divided by a quality factor $Q$ along the path, and is a slowly varying function of distance and frequency. Acceleration spectra with this type of attenuation can be found in Fig. 4. We can evaluate the total-power as

$$
P_{a}^{\prime}\left(r_{0}\right)=g(\chi)\left(\frac{M_{0}}{4 \pi \rho r_{0} \beta^{3}}\right)^{2} \frac{\left\langle\Delta \sigma^{2}\right\rangle}{\Delta \sigma_{0}^{2}} \frac{\lambda^{2} \bar{v}^{2}}{L^{2}}\left(\frac{1}{t^{*}}-\frac{\pi \lambda}{2}\right) .
$$

Comparing the total power in Eq. (3.2) and the above expression, a relationship is obtained

$$
\omega_{m}=1 / t^{*} \quad\left(\omega_{m} \gg \lambda\right) .
$$

This proves the understanding for the empirical cutoff frequency of acceleration spectra proposed by Hanks and McGuire (1981).

Extending the total power of short-period acceleration in the case of bilateral faulting, Koyama and Zheng (1991) derived the short-period seismic directivity effect. The short-period seismic directivity effect is for random-phase short-waves, and is independent of seismic Doppler effect for long-waves which is known as the seismic directivity. They investigated the former directivity effect from the azimuthal amplitude variation of strong ground motions by large shallow earthquakes and from the isoseismal distributions of disastrous shallow earthquakes in Japan and China.

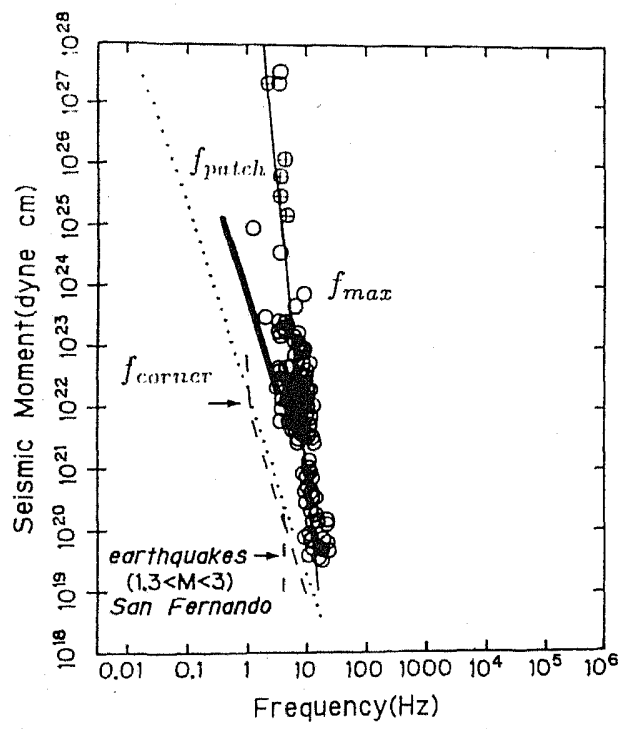

Fig. 5. Corner frequency $f_{\text {corner, }}$ patch corner frequency $f_{\text {patch, }}$ and cutoff frequency $f_{\max }$ versus seismic moment. Dotted line is for $f_{\text {corner }}$ to $M_{o}$ relation derived from a similarity rule for large earthquakes. Thick line is for $f_{\text {patch }}$ to $M_{o}$ relation (Koyama, 1985) and $f_{\max }$ estimates (Aki, 1989) are plotted by circles. 


\section{$3.2 f_{\max }$ and self-similar fault patches}

Figure 5 summarizes corner frequency $f_{\text {corner }}$, patch corner frequency $f_{\text {patch }}$, and cutoff frequency $f_{\max }$ thus far obtained (Aki, 1989; Koyama, 1985). Frequency $f(\mathrm{~Hz})$ is used here but other frequencies are still angular frequency. Most of the data points, obtained from moderate-size earthquakes, are in a frequency range where three characteristic frequencies merge. It appears that $f_{\max }$ estimation for large earthquakes changes slightly with respect to seismic moment, though the number of the data is very small to reach a definite conclusion. Let us consider the physical basis of the cutoff frequency $f_{\max }$ in more detail.

There are three hypotheses in considering the theory of cutoff frequency $f_{\max }:$ In the first hypothesis, we have studied previously in Sec. 3.1 that the cutoff frequency $\omega_{m}$ is attributed to inelastic attenuation, where

$$
\omega_{m}=2 \pi f_{\max }=1 / t^{*} \text {. }
$$

This hypothesis leads us to a constant $f_{\max }$ irrespective of the earthquake source sizes. There would not be any portion of the white acceleration spectrum for some earthquakes, when the corner frequency and the cutoff frequency are of the same order. As an example, we see in Fig. 4(b) where a strong attenuation controls the spectral behavior in high frequencies without any portion of a constant acceleration spectrum.

The second hypothesis is based on the dynamics of crack-tip propagation. Consider a shear crack extending at a velocity $\bar{v}$. A stress concentration appears at the front of the crack tip. The stress concentration is inversely proportional to the square root of the distance from the crack tip. Such stress singularity is physically unrealistic. A cohesive zone behind the crack tip has been proposed to remove this stress singularity (Rice, 1980). The existence of a cohesive force decelerates the propagation velocity of the rupture front, and also smooths the seismic impulse emitted at the instants of onset and arrest of the rupture. A time constant for the smoothing can be estimated from the breakdown of the cohesive zone. This smoothing effect has been considered as the origin of $f_{\max }$ by Papageorgiou and Aki (1983). This $f_{\max }$ hypothesis is presumed to be

$$
f_{\max } \simeq \bar{v} / \bar{c},
$$

where $\bar{c}$ is the cohesive zone size. In other words, this time constant $\bar{c} / \bar{v}$ is a rise time for the stress drop to build up from the static to the dynamic friction level at the crack tip.

The characteristic size of cohesive zones must be very small compared to the fault length and width of large earthquakes. Once the rupture propagates to a sufficient distance, the size of the cohesive zone would depend only on the fault segment in the vicinity of the rupture front (crack tip). This suggests that the time constant $\bar{c} / \bar{v}$ is very small and that the $\delta$-function like time variation of the stress drop is for the earthquake faulting. As a consequence, this hypothesis also implies a constant cutoff frequency for the acceleration spectra of large earthquakes.

The third hypothesis to account for the cutoff frequency $f_{\max }$ is obtained by a scaling relation to the size-number distribution of random fault patches. Let us consider the minimum characteristic corner frequency $\zeta_{1}$ from the rupture of the largest fault patch. Suppose that there are $j$-times more fault patches than the largest fault patch, which have a characteristic corner frequency $k$-times larger than $\zeta_{1}$. The same procedure

Vol. 42, No. 2, 1994 
is applied to obtain $j^{2}$-times more fault patches specified by $k^{2}$-times larger characteristic corner frequency. This scaling is repeated by $M$-steps up to the largest characteristic frequency $\zeta_{2}$. Then there would be a self-similar set of random fault patches on the heterogeneous fault. The largest fault patch and the smallest fault patches specify the scaling region where the scaling relation is applicable. The acceleration spectrum can be expressed by the discrete sum of squared acceleration spectra of self-similar fault patches:

$$
\begin{aligned}
G_{f}(P, S)= & \frac{M_{o}\left|R_{\theta \phi}(P, S)\right|}{4 \pi \rho r_{0}\left(\alpha^{3}, \beta^{3}\right)} \frac{\omega^{2}\left|\sin \left\{\left(k_{c}-\omega / \bar{v}\right) L / 2\right\}\right|}{\left|\left(k_{c}-\omega / \bar{v}\right) L / 2\right|} \\
& \times\left[\frac{\sin ^{2}\left(\omega T_{0} / 2\right)}{\left(\omega T_{0} / 2\right)^{2}}+\frac{2 \zeta_{1} \sigma^{2}}{T_{0} \bar{a}^{2}} \frac{1-(j / k)}{1-(j / k)^{M+1}} \sum_{m=0}^{M} \frac{j^{m}}{\omega^{2}+\left(\zeta_{1} k^{m}\right)^{2}}\right]^{1 / 2},
\end{aligned}
$$

where $M$ specifies the level of the scaling, and the maximum characteristic corner frequency $\zeta_{2}$ is $\zeta_{1} k^{M}$ for $j^{M}$ pieces of fault patches.

Figure 4 shows normalized acceleration spectra of $G_{f}(S)$, taking parameters of $j=2, k=3$ for the spectrum labeled by Cantor and $j=4, k=3$ for that by Koch. These acceleration spectra are more complicated than the stochastic model in Eq. (3.1) also shown in Fig. 4. The spectral behavior is represented by a frequency-dependence of $\omega^{2}-\omega^{0}-\omega^{2-\delta}-\omega^{\kappa / 2}-\omega^{0}$ with increasing frequency. The power coefficient $\kappa$ relates to a fractal dimension of the self-similar fault patches and is defined as (Koyama and Hara, 1992),

$$
\kappa=\ln j / \ln k .
$$

This spectral behavior reveals the existence of three corner frequencies: standard corner frequency from the rupture duration of the entire faulting, two characteristic corner frequencies $\zeta_{1}$ and $\zeta_{2}$ resulting from the rupture process of the largest and smallest fault patches. The second corner frequency $\zeta_{1}$ should correspond to the patch corner frequency in these fractal fault-patch models of Cantor and Koch.

Figure 4 (a) and (b) also show acceleration spectra for small attenuation and large attenuation, respectively. High-frequency spectra are strongly distorted by the attenuation effect in Fig. 4(b). In Fig. 4(a), we find that the third corner frequency $\zeta_{2}$ of the Cantor and the Koch spectra gives a sharp cutoff in the high-frequency acceleration spectrum. If we consider that this corner frequency corresponds to $f_{\max }$, then the third hypothesis proposes a source-oriented $f_{\max }$. This hypothesis describes $f_{\max }$ as the rupture duration of the smallest fault patches on the heterogeneous fault.

In summary, the third hypothesis proposes the source-oriented cutoff frequency $f_{\max }$, and also describes the spectral behavior of high-frequency acceleration in relation to the size distribution of fault heterogeneities. The origin of the cutoff frequency $f_{\max }$ has not been settled by these three hypotheses, because direct measurements of such cutoff frequencies have been made for only a small number of earthquakes under the band-limited observation. A scaling law relevant to the cutoff frequency, i.e., how the frequency changes with respect to the earthquake source size, is not yet completely understood. 


\section{Seismic Energy of Complex Faulting Process}

Energy is the most fundamental quantity that measures the strength of earthquake sources. There are basically three different methods to estimate the energy of earthquakes: The first method is an empirical estimate by calculating the seismic energy $E_{S}$ from surface-wave magnitude $M_{S}{ }^{*}$ using the Gutenberg and Richter's relation (1954):

$$
\log E_{S}=1.5 M_{S}+11.8 \text {. }
$$

The physical significance of this method is somewhat uncertain. The second method is a static estimate to obtain the seismic energy from seismic moment $M_{o}$, stress drop $\Delta \sigma_{0}$, and fault dimension $L W$. This gives the energy available for the seismic radiation from the faulting process. The third method is a kinetic estimate of the seismic-wave energy.

Theoretically, the kinetic estimate of seismic-wave energy requires the knowledge of a space-time history of the faulting process. On the other hand, the static estimate requires only static parameters of the fault process irrespective of the time history of an earthquake source. These two representations are independent of each other and have been believed to specify the dynamical boundary condition on the faulting process (Kanamori, 1977; Savage and Wood, 1971).

The estimate of seismic energy of earthquakes is usually made under an implicit assumption of the smooth-and-coherent faulting process. The recent advance in seismometry enables us to calculated seismic-wave energy of large earthquakes from broad-band seismic waves. Their results can not be directly applied to understand the complex faulting process of heterogeneous earthquakes, since there is no fundamental theory to link the observations and the heterogeneous earthquake sources. In the following, we will derive theoretical expressions for a static energy and a kinetic seismic-energy of the complex faulting process.

\subsection{Static estimate of seismic energy of heterogeneous faulting}

Let stress drop $\Delta \sigma(\vec{\xi})$ and associated dislocation $D(\vec{\xi})$ be at a point $\vec{\xi}$ on a fault area $S$. An average stress drop is $\Delta \sigma_{0}$ and the average dislocation is $D_{0}$ on the fault plane. Seismic moment $M_{o}$ in this case is expressed as:

$$
M_{o}=\mu \int_{S} D(\vec{\xi}) \mathrm{d} S=\mu D_{0} S .
$$

Consider a simple model of an earthquake source where $\sigma_{1}(\vec{\xi}), \sigma_{2}(\vec{\xi})$, and $\sigma_{f}(\vec{\xi})$ are the initial, final, and frictional stresses at a point $\vec{\xi}$ on the fault. Then the energy available for seismic waves is given by Savage and Wood (1971) as

$$
W_{0}=\int_{S}\left\{\frac{\sigma_{1}(\vec{\xi})+\sigma_{2}(\vec{\xi})}{2}-\sigma_{f}(\vec{\xi})\right\} D(\vec{\xi}) \mathrm{d} S .
$$

* $M_{S}$ is the surface-wave magnitude defined by Gutenberg (1945). Recent surface-wave magnitude $M_{s}$ is slightly different from $M_{S}$, because of the historical change in seismometry. We did not pay any attention to this difference in the following analysis, because the difference is as small as 0.2 (Abe, 1981).

Vol. 42, No. 2, 1994 
The first term in the integrand indicates the total work done by the stress drop on the fault, and the second is the energy dissipated by the friction. Since the local stress drop is

$$
\Delta \sigma(\vec{\xi})=\sigma_{1}(\vec{\xi})-\sigma_{2}(\vec{\xi})
$$

we can rewrite Eq. (4.3) as

$$
W_{0}=\int_{S}\left\{\frac{\Delta \sigma(\vec{\xi})}{2}-\left(\sigma_{f}(\vec{\xi})-\sigma_{2}(\vec{\xi})\right)\right\} D(\vec{\xi}) \mathrm{d} S .
$$

The Orowan's condition (1960) is considered to make the discussion simple, where the fault motion stops when the stress decreases to the frictional stress:

$$
\sigma_{f}=\sigma_{2} .
$$

When $D(\vec{\xi})=D_{0}$ and $\Delta \sigma(\vec{\xi})=\Delta \sigma_{0}$ all over the fault plane, the energy available for the seismic-wave radiation in Eq. (4.5) is approximated as

$$
\bar{W}_{0}=\Delta \sigma_{0} M_{o} / 2 \mu \text {. }
$$

Kanamori (1977) used this relationship to estimate the energy released by great shallow earthquakes. With typical values of $\Delta \sigma_{0}=30 \mathrm{bar}$, and $\mu=10^{11} \mathrm{dyne} / \mathrm{cm}^{2}$,

$$
\bar{W}_{0} \simeq\left(4 \times 10^{-5}\right) M_{o} .
$$

The Gutenberg and Richter's energy estimate in Eq. (4.1) agrees fairly well with the above result in Eq. (4.8) (Kanamori and Anderson, 1975).

When the stress drop on the fault is no longer constant, the theory is not as simple as Eq. (4.7). Since the stress drop and dislocation are fluctuating locally on the fault plane, they.are approximated by the sum of a deterministic part of the faulting and a stochastic part of random fault patches as

$$
\begin{aligned}
& \Delta \sigma(\vec{\xi})=\Delta \sigma_{0}+\delta \sigma(\vec{\xi}), \\
& D(\vec{\xi})=D_{0}+\delta D(\vec{\xi}),
\end{aligned}
$$

where $\delta \sigma$ and $\delta D$ are the random fluctuation of local stress drop and local dislocation, respectively, and they are characterized by zero mean. In this case, the energy $W_{0}$ in Eq. (4.5) is rewritten as

$$
W_{h}=\bar{W}_{0}+\frac{1}{2} \iint_{S} \delta \sigma(\vec{\xi}) \delta D(\vec{\xi}) \mathrm{d} S,
$$

where the Orowan's condition in Eq. (4.6) is considered. The second term in the righthand side of Eq. (4.11) results from the random fault patches. It is reasonable that the displacement on a fault patch is large where the fluctuation of stress drop is positive and that it is small where the fluctuation is negative. In this way, the second term would not vanish.

The Cauchy-Schwarz inequality yields the estimate of the work done by the stochastic part of the complex faulting process (Appendix A). Then the upper bound of the energy available for seismic-waves from the complex faulting process is from 
Eqs. (4.7) and (A.7)

$$
W_{h}=\frac{\Delta \sigma_{0}}{2 \mu} M_{o}\left\{1+\frac{\left\langle\Delta \sigma^{2}\right\rangle}{\Delta \sigma_{0}^{2}}\left(\frac{\lambda T_{0}}{2}\right)^{1 / 2}\right\} .
$$

The above representation reveals the seismic energy from the complex faulting process.

\subsection{Kinetic estimate of seismic-wave energy of heterogeneous faulting}

The seismic-wave energy can be evaluated from observed body-waves (e.g., Anderson et al., 1986; Boatwright and Choy, 1986; Kikuchi and Fukao, 1988; Vassiliou and Kanamori, 1982). The general form by Haskell (1964) is further extended to formulate a seismic-wave energy radiated from the complex faulting process:

$$
E_{S}=\rho \int_{-\infty}^{\infty} \int_{0}^{2 \pi} \int_{0}^{\pi}\left\{\alpha \dot{u}_{r}^{2}+\beta\left(\dot{u}_{\theta}^{2}+\dot{u}_{\phi}^{2}\right)\right\} \mathrm{d} t r_{0}{ }^{2} \sin \theta \mathrm{d} \theta \mathrm{d} \phi,
$$

where $\rho$ is density, and $\alpha$ and $\beta$ are $\mathrm{P}$ - and S-wave velocities, respectively. Displacement components in Eqs. (2.1) and (2.2) are applied to evaluate the theoretical P- and S-wave energies. The time-domain integral in Eq. (4.13) is converted to the frequency domain by Parseval's equality. A lengthy but straightforward integration yields

$$
\frac{1}{2 \pi} \int_{-\infty}^{\infty} \hat{\dot{u}}_{c}(\omega)^{2} \mathrm{~d} \omega \simeq \frac{1}{2 \pi}\left(\frac{M_{o}\left|R_{\theta \phi}^{c}\right|}{4 \pi \rho r_{0} c^{3}}\right)^{2}\left[\frac{\omega_{c}^{2}}{2 T_{0}}+\frac{\lambda\left\langle\Delta \sigma^{2}\right\rangle}{4 \Delta \sigma_{0}{ }^{2}} \omega_{c}^{2}\right],
$$

where $\hat{\dot{u}}$ represents the Fourier transform of $\dot{u}$, and $\lambda>\omega_{c}$ is assumed, where $\lambda$ is the patch corner frequency defined in Eq. (2.6) and $\omega_{c}$ is the corner frequency in Eq. (2.11).

The integration of Eq. (4.13) by $\theta$ and $\phi$ is now straightforward. Consequently, $\mathrm{P}$-wave energy radiated from the complex faulting process is

$$
E_{S}^{P}=\rho \alpha \frac{2 \bar{v}^{2} M_{o}^{2}}{\pi \rho^{2} \alpha^{6} L^{2}}\left[\frac{1}{2 T_{0}}+\frac{\lambda\left\langle\Delta \sigma^{2}\right\rangle}{4 \Delta \sigma_{0}^{2}}\right]\left[\frac{2}{3 v^{2}}-\frac{4}{v^{4}}+\left(\frac{1}{v^{3}}-\frac{2}{v^{5}}\right) \ln \left|\frac{1-v}{1+v}\right|\right],
$$

where $v=\bar{v} / \alpha$, and $S$-wave energy is

$$
E_{S}^{S}=\rho \beta \frac{\bar{v}^{2} M_{o}^{2}}{2 \pi \rho^{2} \beta^{6} L^{2}}\left[\frac{1}{2 T_{0}}+\frac{\lambda\left\langle\Delta \sigma^{2}\right\rangle}{4 \Delta \sigma_{0}^{2}}\right]\left[\frac{8 \chi^{4}-50 \chi^{2}+48}{3 \chi^{4}\left(1-\chi^{2}\right)}+\frac{8-3 \chi^{2}}{\chi^{5}} \ln \left|\frac{1-\chi}{1+\chi}\right|\right],
$$

where $\chi=\bar{v} / \beta$. The net seismic-wave energy $E_{S}$ is then

$$
E_{S}=E_{S}^{P}+E_{S}^{S} \text {. }
$$

The seismic-wave energy in Eq. (4.17) is identical to the result by Haskell (1964) when the faulting is smooth-and-coherent without heterogeneities, that is, $\left\langle\Delta \sigma^{2}\right\rangle=0$.

These theoretical representations are the fundamental expressions to make a bridge connecting the complex faulting process of large earthquakes and the calculated seismic energy from the recent digital seismometry.

The energy ratio of S- to $\mathrm{P}$-waves is strongly dependent on the mode of earthquake

Vol. 42, No. 2, 1994 
faultings. A point double-couple gives a ratio of about 23.4 (Honda, 1962), and a circular faulting model by Sato and Hirasawa (1973) gives the ratio of about 20. A coherent rupture propagating on a rectangular fault yields a ratio of about 30 to 50 with respect to the rupture propagation velocity (Haskell, 1964).

The P- and S-wave energy representations in Eqs. (4.15) and (4.16) predict an energy ratio of about $35,47,54$, and 60 , when $\left\langle\Delta \sigma^{2}\right\rangle / \Delta \sigma_{0}^{2}$ is $0,1 / \lambda T_{0}, 2 / \lambda T_{0}$, and $4 / \lambda T_{0}$. Corresponding value of $\sigma^{2} / \bar{a}^{2}$ is $0,0.5,1.0$, and 2.0 , respectively. In this calculation, $L=W, \bar{v} / \beta=0.83$ and $\lambda /(W / \beta)=200$ are assumed. The first case of $\left\langle\Delta \sigma^{2}\right\rangle / \Delta \sigma_{0}^{2}=0$ is for the deterministic source without any fault heterogeneities. It is understood that the complex faulting process radiates much more S-wave energy than the other source models.

\subsection{Seismic efficiency of heterogeneous faulting}

Seismic efficiency is defined by a ratio of seismic-wave energy and total work done by an earthquake. That is

$$
\eta_{0}=E_{S} / W_{t}
$$

where $E_{S}$ is the seismic-wave energy and $W_{t}$ is the total work done by an earthquake. The total work is not the same as the energy available for seismic waves in Eq. (4.3). It is defined

$$
W_{t}=\int_{S} \frac{\sigma_{1}(\vec{\xi})+\sigma_{2}(\vec{\xi})}{2} D(\vec{\xi}) \mathrm{d} S,
$$

where $S$ is an earthquake source size. If an earthquake faulting is smooth and coherent, the seismic efficiency is

$$
\eta_{0}=\left\{\left(\bar{\sigma}_{1}-\bar{\sigma}_{2}\right)-2\left(\bar{\sigma}_{f}-\bar{\sigma}_{2}\right)\right\} /\left(\bar{\sigma}_{1}+\bar{\sigma}_{2}\right)
$$

where $\bar{\sigma}_{1}, \bar{\sigma}_{2}$, and $\bar{\sigma}_{f}$ are the average values of initial, final and frictional stresses on the fault.

When an earthquake faulting is irregular, stress drop and dislocation on a fault change from place to place. The total work Eq. (4.19) by the complex faulting process is

$$
W_{t} \simeq \frac{\Delta \sigma_{0}}{2 \mu} M_{o}\left\{\frac{\bar{\sigma}_{1}+\bar{\sigma}_{2}}{\Delta \sigma_{0}}+\frac{\left\langle\Delta \sigma^{2}\right\rangle}{\Delta \sigma_{0}^{2}}\left(\frac{\lambda T_{0}}{2}\right)^{1 / 2}\right\} .
$$

This can be derived similarly as Eq. (4.12) using Eqs. (4.3) and (4.11).

Since S-wave energy is dominant, the seismic-wave energy radiated from the complex faulting process can be approximated by the S-wave energy of Eq. (4.16). Then the seismic efficiency of the complex faulting process is expressed approximately as

$$
\eta_{h}=\frac{p}{4 q}\left\{1+\frac{\left\langle\Delta \sigma^{2}\right\rangle}{\Delta \sigma_{0}{ }^{2}} \frac{\lambda T_{0}}{2}\right\} \chi^{3} g(\chi) /\left\{\frac{\bar{\sigma}_{1}+\bar{\sigma}_{2}}{\Delta \sigma_{0}}+\frac{\left\langle\Delta \sigma^{2}\right\rangle}{\Delta \sigma_{0}{ }^{2}}\left(\frac{\lambda T_{0}}{2}\right)^{1 / 2}\right\},
$$

where $g(\chi)$ is in Eq. (3.3). The important effect of the fault heterogeneities on the seismic efficiency will be discussed again in Sec. 6 . 


\section{Earthquake Magnitude and Complex Faulting Process}

Earthquake magnitude has been evaluated for each earthquake to estimate its strength and energy since the end of the last century (Abe, 1981; Geller and Kanamori, 1977; Gutenberg and Richter, 1954; Utsu, 1982a). The magnitude of recent local earthquakes has been also determined by a variety of methods using different kinds of data (Adams, 1977; Lee and Wetmiller, 1978). The recent trend in seismology is to describe the earthquake in terms of source parameters discussed previously. However, the earthquake magnitude is a parameter widely used to measure both large and small earthquakes in the world.

We will study the earthquake magnitude, considering the long-period and the short-period approximations of the complex faulting process. The present study would not make the same approach as previous studies such as Aki (1972), Bäth (1981), Geller (1976), Gusev (1991), Kanamori and Anderson (1975), Koyama et al. (1982), Noguchi and Abe (1977), and Utsu (1982b). An implicit assumption has been employed in the previous studies, where the time-domain amplitude is proportional to the earthquake source spectrum, which is inadequate. We will study the earthquake magnitude in more detail based on its original definition, considering the band-limited nature of seismic waves. The relationship between the time-domain amplitude and the spectral component will be presented from this new standpoint.

\subsection{Long-wavelength description of surface-wave magnitude}

The definition of surface-wave magnitude states that $M_{S}$ is a logarithm of the maximum amplitude of dominant surface waves with a period of about $20 \mathrm{~s}$, after correcting for the wave-travel distance (Gutenberg, 1945). The wavelength of 20-s surface waves would be about 60 to $80 \mathrm{~km}$. This suggests that $M_{S}$ is a measure of earthquake sources as a long-wavelength description for those earthquakes smaller than $60-80 \mathrm{~km}$ in the characteristic fault dimension. It should be understood that $M_{S}$ would be a measure as a short-wavelength description for great earthquakes with fault dimension much larger than $80 \mathrm{~km}$. An earthquake of 60 to $80 \mathrm{~km}$ fault-length is graded by $M_{S}$ of about 7.5 .

Reminding the source spectrum in Eq. (2.8), the low-frequency approximation gives the source spectral density in proportion to $\left(L W T_{0} \vec{a}\right)$. Usually the spectrum in this low-frequency range is obtained from long waves with continuous phase. Waves with continuous phase are amplitude-additive. Generally speaking, the time-domain amplitude in a particular frequency band after being multiplied by the time duration of its wave train is related to the corresponding spectral component. This derivation is given in Appendix B. It is assumed here that time $L / \bar{v}$ represents the time duration of the wave train. If the time duration is much shorter than $20 \mathrm{~s}$, the time-domain amplitude $A_{\max }$ of continuous long-waves, after correcting for the attenuation due to wave travel-distance and inelasticity, is expressed as Eq. (B.5) in Appendix B

$$
A_{\max }=c_{0} M_{o} / 2 \pi \mu \text {, }
$$

where $c_{0}$ is a constant which is independent of the earthquake source size. This is valid for small earthquakes, since the time duration is assumed to be small. For large

Vol. 42, No. 2, 1994 
earthquakes, the amplitude is approximated by the seismic moment divided by the time duration as in Eq. (B.7) in Appendix B

$$
A_{\max }=c_{0} M_{o} \bar{v} / \mu L \text {. }
$$

Since $A_{\max }$ is the maximum amplitude in the time domain, the logarithm of $A_{\max }$ should represent a theoretical surface-wave magnitude $M_{S}$ as

$$
M_{S}=\log M_{o}-\log (2 \pi \mu)+\epsilon,
$$

for small earthquakes from Eq. (5.1), and from Eq. (5.2)

$$
M_{S}=\log \left(M_{o} / L\right)+\log (\tilde{v} / \mu)+\epsilon,
$$

for large earthquakes. Here $\epsilon$ is a constant depending on the magnitude definition and the unknown constant $c_{0}$. The assumption of $L / \bar{v}$ employed for the time duration is heuristic and it should be justified from empirical observations, however, the term $\epsilon$ will compensate for the defect of this assumption. The representations of surface-wave magnitude in Eqs. (5.3) and (5.4) more rigorously follow the definition of the magnitude scale.

A relationship between seismic moment and surface-wave magnitude for large earthquakes can be derived from Eqs. (5.4) and (2.13), applying the similarity rules,

$$
\log M_{o}=\frac{3}{2} M_{S}-\frac{1}{2} \log \left(\frac{p^{2} \Delta \sigma_{0} \bar{v}^{3}}{\mu^{3}}\right)-\frac{3}{2} \epsilon .
$$

Similarly, when the fault dimension $S$ is approximated to be $L W$, the following theoretical relationship for $M_{S}$ and $S$ for large earthquakes is derived

$$
\log S=M_{S}-\log (p q \bar{a})-\epsilon .
$$

The relationship between $M_{S}$ and $M_{o}$ in Eq. (5.3) is very important, because it reveals the meaning of an empirical relation by Ekström and Dziewonski (1988),

$$
\log M_{o}=M_{s}+19.24 \quad M_{s}<5.3,
$$

where the unit of $M_{o}$ is dyne $\cdot \mathrm{cm}$. Combining the theoretical representation in Eq. (5.3) and the empirical relation in Eq. (5.7), a constraint is obtained

$$
\log (2 \pi \mu)-\epsilon=19.24 \quad M_{s}<5.3 .
$$

Due to the unit of $M_{o}$, the rigidity $\mu$ has the unit of dyne $\cdot \mathrm{cm}^{-2}$, and all the other parameters are in c.g.s. unit. An empirical relation by Ekström and Dziewonski (1988) and Purcaru and Berckhemer (1978) is also known for large earthquakes

$$
\log M_{o}=1.5 M_{s}+16.14 \text {. }
$$

Comparing the above relation with the theoretical relationship in Eq. (5.5), another constraint is obtained

$$
\log \left(\frac{p^{2} \Delta \sigma_{0} \bar{v}^{3}}{\mu^{3}}\right)+3 \epsilon=-32.28
$$


Furthermore, an empirical relation between fault dimension and magnitude has been obtained by Utsu and Seki (1954):

$$
\log S=1.02 M-4.01,
$$

where $S$ is mostly aftershock areas of large earthquakes in and near Japan in the unit of $\mathrm{km}^{2}$. Originally, $M$ in Eq. (5.11) is an earthquake magnitude by the Japan Meteorological Agency, and it has been calibrated against $M_{S}$ (Utsu, 1982b). Considering the theoretical relationship in Eq. (5.6), the above empirical relation in Eq. (5.11) is reduced to

$$
\log (p q \bar{a})+\epsilon=-6.0 \text {. }
$$

This indicates that the empirical relation in Eq. (5.11) represents the kinematical similarity of $\bar{a}$. We note rounding-off small quantities and c.g.s. unit in this last relation.

As a consequence, these empirical relations are understood to be the similarity rules among macroscopic source parameters of the complex faulting process. It is worth noting that these understandings are based on the long-wavelength description of the $M_{S}$ determination.

\subsection{Short-wavelength description of surface-wave magnitude}

The empirical relation in Eq. (5.11) between fault dimension $S$ and magnitude $M_{S}$ fits the data of large earthquakes when $M_{S}$ is smaller than about 7 . However, there are systematic deviations of the great earthquake data from the relation. Figure 6 shows

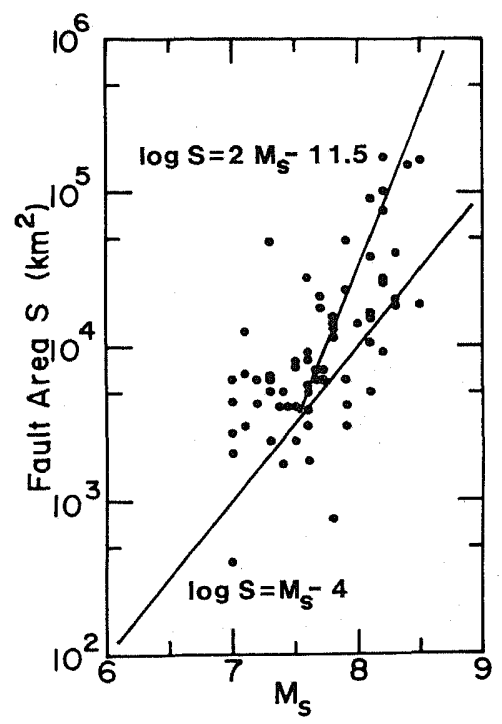

Fig. 6. Relation between surface-wave magnitude $M_{S}$ and earthquake source dimension $S$ (Koyama and Shimada, 1985). An empirical relation of $\log S=$ $M_{S}-4$ is equivalent to Utsu and Seki's (1954) relation for earthquakes in and near Japan. The other relation of $\log S=2 M_{S}-11.5$ is for great earthquakes in the world.

Vol. 42, No. 2, 1994 


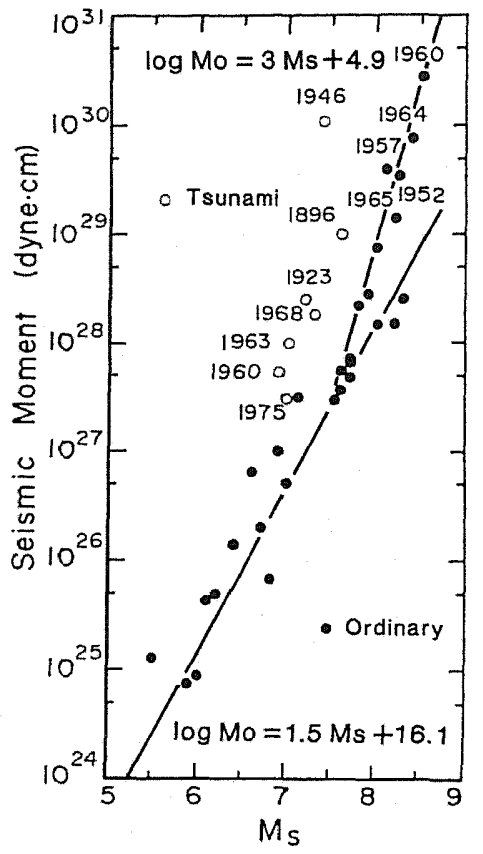

Fig. 7. Seismic moment $M_{o}$ and surface-wave magnitude $M_{S}$ for large and great earthquakes (Takemura and Koyama, 1983). Solid and open circles indicate ordinary and tsunami (low-frequency) earthquakes, respectively. Note that large tsunami earthquakes show a tendency between $M_{o}$ and $M_{S}$ which is similar to great earthquakes in the world.

$M_{S}$ and $S$ for great earthquakes. Another empirical relation has been obtained for these data (Koyama and Shimada, 1985):

$$
\log S=2 M_{S}-11.5,
$$

where $S$ is in the unit of $\mathrm{km}^{2}$. This relation reasonably explains the scattered data when $M_{S}$ is larger than 7.5. In this case, the long-period/low-frequency approximation is no longer appropriate for $M_{S}$ determination.

A similar observation can be seen in the $M_{o}$ versus $M_{S}$ relation. Figure 7 summarizes $M_{o}$ and $M_{S}$ for large and great earthquakes. The 1960 Chilean, 1964 Alaskan, 1957 Aleutian, 1952 Kamchatkan, and the 1965 Rat Island earthquakes are the greatest, and deviate systematically from the previous $M_{o}-M_{S}$ relation of Eq. (5.9). Another empirical relation has been proposed to explain these data by Takemura and Koyama (1983):

$$
\log M_{o}=3 M_{S}+4.9 \text {. }
$$

It seems that the data set is too small to definitely confirm this relation, however, the same tendency can be found from the recent independent data by Ekström and Dziewonski (1988). 


\subsubsection{Previous model by destructive interference}

Let us now consider one of the high-frequency approximation of the source spectrum in Eq. (2.8), where there is no heterogeneity, $\sigma^{2}=0$. A particular frequency $\omega_{s}$ for the $M_{s}$ determination is $2 \pi / 20$. Consider the case when $\omega_{s}$ is much larger than $\omega_{c}$ but smaller than $2 / T_{0}$, where $\omega_{c}$ and $2 / T_{0}$ are the characteristic frequencies of the source spectrum in Eq. (2.8); this is when an approximation of $\left|\sin \left(\omega_{s} / \omega_{c}\right) /\left(\omega_{s} / \omega_{c}\right)\right| \simeq$ $1 /\left|\omega_{s} / \omega_{c}\right|$ is valid. Since $\omega_{c}$ in Eq. (2.11) can be approximated as $2 \bar{v} / L$, the source spectrum at $\omega_{s}$ reduces to $M_{o} /\left(\mu \omega_{s} L / 2 \vec{v}\right)$. In this case, the time domain amplitude $A_{\max }$ is expressed similarly as Eq. (5.2):

$$
A_{\max }=c_{0} \frac{2 \bar{v} M_{o}}{\mu \omega_{s} L} \frac{\bar{v}}{L}
$$

where a time duration of wave trains is $L / \bar{v}$ again. By taking a logarithm of the maximum amplitude in the time domain of Eq. (5.15), a magnitude representation for large earthquakes is

$$
\log M_{o}=3 M_{S}-\log \left\{\frac{8 p^{4} q^{2} \bar{a}^{2} \bar{v}^{4}}{\mu \omega_{s}^{3}}\right\}-3 \epsilon \quad\left(\frac{20}{\pi}<\frac{L}{\bar{v}}<\frac{20}{\pi p q}\right) .
$$

This relationship has been applied to study the earthquake source parameters of large earthquakes (e.g., Kanamori and Anderson, 1975). The condition attached to Eq. (5.16) which was derived from the assumption of $\omega_{c}<\omega_{s}<2 / T_{0}$, is reduced to approximately $20<L(\mathrm{~km})<80$, which indicates that the long-wavelength description of $M_{S}$ is appropriate for such earthquakes. This contradicts the basic consideration in this section.

\subsubsection{Scaling patch corner frequency model}

A theoretical representation for surface-wave magnitude is derived by the short-period approximation of the complex faulting process: The rise time $T_{0}$ increases in accordance with the size of the earthquake. $T_{0}$ of great earthquakes would become large enough to satisfy the condition in Eq. (2.8):

$$
\left(\frac{2}{\omega_{s} T_{0}}\right)^{2} \ll \frac{2 \sigma^{2}}{T_{0}{ }^{2} \bar{a}^{2}} \frac{\lambda T_{0}}{\omega_{s}^{2}+\lambda^{2}} .
$$

In this case, 20-s surface waves are excited mostly by the rupture of random fault patches. Since surface waves in this case are characterized as short waves, the phase of waves is random. Amplitude-additive spectral analysis is no longer appropriate for such waves. We should perform an energy-additive spectral analysis in this case. A theory is developed in Appendix $C$ to account for the relationship between time-domain amplitude and spectral amplitude of random-phase short-waves. An rms amplitude of random-phase seismic waves at $\omega_{s}$ can be expressed (Appendix C)

$$
A_{\mathrm{rms}} \simeq c_{0}\left\{\frac{1}{\pi}\left(\frac{2 \vec{v} M_{o}}{\mu \omega_{s} L}\right)^{2}\left[\frac{2 \sigma^{2}}{T_{0}{ }^{2} \bar{a}} \frac{\lambda T_{0}}{\omega_{s}{ }^{2}+\lambda^{2}}\right] \frac{\bar{v}}{L}\right\}^{1 / 2},
$$

where a time duration of $L / \bar{v}$ is assumed also for random-phase short-waves. The constant $c_{0}$ is again introduced, because it would not depend on the earthquake source size.

Vol. 42, No. 2, 1994 
Neither a scaling rule nor an absolute value of patch corner frequency $\lambda$ is known for large and great earthquakes. To extend the theory of $M_{S}$ for incoherent short waves, two models for the patch corner frequency are considered: The first model is the same as Eq. (2.22) where $\lambda$ is scaled by the corner frequency even for great earthquakes. This model has been termed the scaling P-model. Figure 5 suggests $\omega_{s}{ }^{2}+\lambda^{2} \simeq 2 \omega_{s}{ }^{2}$ for large and great earthquakes in this context, since an extrapolation of patch corner frequency gives $f_{p}(=\lambda / 2 \pi) \simeq 0.05 \mathrm{~Hz}$ for $M_{o}=10^{28}$ dyne $\cdot \mathrm{cm}$. Then, the maximum amplitude of random-phase short-waves is expressed in terms of the rms amplitude in Eq. (5.18) as

$$
A_{\max } \simeq \frac{2 c_{0} \bar{v}}{\omega_{s}{ }^{2}}\left(\frac{p q \lambda \sigma^{2}}{\pi}\right)^{1 / 2} W f(N),
$$

where $f(N)$ is a function of the number $N$ of peaks and troughs of short waves. Applying the statistical theory of extremes (Boore, 1983; Koyama and Shimada, 1985), the function of $f(N)$ is derived

$$
f(N)=(2 \ln N)^{1 / 2}+\gamma /(2 \ln N)^{1 / 2},
$$

where $\gamma$ is the Euler constant. A Rayleigh distribution is assumed for peak and trough amplitudes in this case. Another expression is

$$
f(N)=\left\{\ln \left(N^{2} / 2 \pi\right)-\ln \ln \left(N^{2} / 2 \pi\right)\right\}^{1 / 2},
$$

where a Gaussian distribution is assumed for peak and trough amplitudes. Roughly speaking, the characteristic frequency of a box car time function with a time duration of $L / \bar{v}$ is about $1 / \omega_{\beta}$, the number $N$ could be approximated similarly as Hanks and McGuire (1981)

$$
N=2 \omega_{s} / \omega_{\beta} .
$$

\subsubsection{Constant patch corner frequency model}

The second model assumes a constant patch corner frequency for great earthquakes (constant P-model). Since Fig. 5 indicates that $f_{p}(=\lambda / 2 \pi)$ is as small as $1 \mathrm{~Hz}$ for small and moderate-size earthquakes, $\lambda=2 \pi$ is tentatively assumed (this will be redetermined more specifically in Sec. 6). In this case, a condition of $\omega_{s}^{2} \ll \lambda^{2}$ is satisfied, since $\omega_{s}$ is $2 \pi / 20$. The rms amplitude in Eq. (5.18) is then rewritten to give the maximum amplitude of random-phase short-waves as

$$
A_{\max } \simeq \frac{2 c_{0} \bar{v}}{\omega_{s}}\left(\frac{2 p q \sigma^{2}}{\pi \lambda}\right)^{1 / 2} W f(N) .
$$

The logarithm of $A_{\max }$ in Eqs. (5.19) and (5.23) should give a measure of $M_{S}$ from random-phase short-waves. Consequently, a theoretical representation of $M_{S}$ for great earthquakes is

$$
M_{S}=\log W+\log \left\{\frac{2 \bar{v}}{\omega_{s}^{2}}\left(\frac{p q \lambda \sigma^{2}}{\pi}\right)^{1 / 2} f(N)\right\}+\varepsilon
$$

for the scaling P-model, and 


$$
M_{S}=\log W+\log \left\{\frac{2 \bar{v}}{\omega_{s}}\left(\frac{2 p q \sigma^{2}}{\pi \lambda}\right)^{1 / 2} f(N)\right\}+\varepsilon
$$

for the constant P-model. A constant $\varepsilon$ may be slightly different from $\epsilon$ in Eq. (5.3). However, it is assumed that $\varepsilon=\dot{\epsilon}$ because of the approximation employed in this analysis and the uncertainty involved in the magnitude determination.

Since $\log S=2 \log W-\log p$, the above theoretical magnitude reduces to show a relationship

$$
\log S=2 M_{S}-2 \log \left\{\frac{2 \vec{v}}{\omega_{s}^{2}}\left(\frac{p q \lambda \sigma^{2}}{\pi}\right)^{1 / 2} f(N)\right\}-\log p-2 \varepsilon
$$

and/or

$$
\log S=2 M_{S}-2 \log \left\{\frac{2 \bar{v}}{\omega_{s}}\left(\frac{2 p q \sigma^{2}}{\pi \lambda}\right)^{1 / 2} f(N)\right\}-\log p-2 \varepsilon
$$

for the scaling and the constant P-models, respectively. These expressions are the short-wavelength description of the complex faulting process to explain the $S-M_{S}$ relation.

Taking the logarithm of the seismic moment, $M_{o}=\Delta \sigma_{0} L W^{2}$, we have

$$
\log M_{o}=3 \log W+\log \left(\frac{\Delta \sigma_{0}}{p}\right) \text {. }
$$

Substituting the theoretical magnitude representations in Eqs. (5.24) and (5.25) into the above equation, a theoretical relationship between $M_{o}$ and $M_{S}$ is also derived based on the short-period approximation of the complex faulting process:

$$
\log M_{o}=3 M_{\mathrm{S}}-3 \log \left\{\frac{2 \bar{v}}{\omega_{s}{ }^{2}}\left(\frac{p q \lambda \sigma^{2}}{\pi}\right)^{1 / 2} f(N)\right\}+\log \left(\frac{\Delta \sigma_{0}}{p}\right)-3 \varepsilon
$$

for the scaling P-model, and

$$
\log M_{o}=3 M_{S}-3 \log \left\{\frac{2 \bar{v}}{\omega_{s}}\left(\frac{2 p q \sigma^{2}}{\pi \lambda}\right)^{1 / 2} f(N)\right\}+\log \left(\frac{\Delta \sigma_{0}}{p}\right)-3 \varepsilon
$$

for the constant P-model.

Comparing the theoretical representations in Eqs. (5.26) and (5.27) and the empirical relation in Eq. (5.13), a constraint is obtained in the c.g.s. unit

$$
\begin{aligned}
& 2 \log \left\{\frac{2 \bar{v}}{\omega_{s}^{2}}\left(\frac{p q \lambda \sigma^{2}}{\pi}\right)^{1 / 2} f(N)\right\}+\log p+2 \varepsilon=1.5, \\
& 2 \log \left\{\frac{2 \bar{v}}{\omega_{s}}\left(\frac{2 p q \sigma^{2}}{\pi \lambda}\right)^{1 / 2} f(N)\right\}+\log p+2 \varepsilon=1.5
\end{aligned}
$$

for the scaling and the constant P-models, respectively. 
Comparing the theoretical representations in Eqs. (5.29) and (5.30) and the empirical relation in Eq. (5.14), another constraint is derived:

$$
\begin{aligned}
& \log \left(\frac{\Delta \sigma_{0}}{p}\right)-3 \log \left\{\frac{2 \bar{v}}{\omega_{s}{ }^{2}}\left(\frac{p q \lambda \sigma^{2}}{\pi}\right)^{1 / 2} f(N)\right\}-3 \varepsilon=4.9, \\
& \log \left(\frac{\Delta \sigma_{0}}{p}\right)-3 \log \left\{\frac{2 \bar{v}}{\omega_{s}}\left(\frac{2 p q \sigma^{2}}{\pi \lambda}\right)^{1 / 2} f(N)\right\}-3 \varepsilon=4.9
\end{aligned}
$$

for the scaling and constant P-models, respectively.

So far, we have discussed the physical basis of surface-wave magnitude. It has also been demonstrated that the wavelength of 20 -s surface waves is the key to understanding the source parameters of the complex faulting process.

\subsection{Short-period description of body-wave magnitude}

It would be timely to investigate body-wave magnitude $m_{b}$ here based on the complex faulting process in order to cross-check the theory developed in this study. Body-wave magnitude $m_{b}$ is calculated from the maximum amplitude of body-waves at about $1 \mathrm{~s} . *$ Therefore, the characteristic frequency $\omega_{b}$ is reasonably assumed to be $2 \pi$. In this frequency, the high-frequency approximation in Eq. (5.17) is always satisfied for earthquakes with $M_{o}$ larger than $10^{20}$ dyne $\cdot \mathrm{cm}$. Koyama and Zheng (1985) showed that body waves of large earthquakes in this period are always complicated and are characterized by random phase. Theoretical representation of $m_{b}$ can be derived in a similar manner as Eq. (5.25):

$$
m_{b}=\log W+\log \left\{f\left(N_{b}\right) \frac{2 \bar{v}}{\omega_{b}}\left(\frac{2 p q \sigma^{2}}{\pi \lambda}\right)^{1 / 2}\right\}+\varepsilon_{b},
$$

where $\omega_{b}=2 \pi$ is used, and $\varepsilon_{b}$ is a constant that depends on the definition of body-wave magnitude and on some constant due to the wave propagation. This representation is for the constant P-model. The expression for the scaling P-model can also be derived.

A relationship between seismic moment and body-wave magnitude can be expressed by substituting the above equation into Eq. (5.28) to yield

$$
\log M_{o}=3 m_{b}-3 \log \left\{\frac{2 \bar{v}}{\omega_{b}}\left(\frac{2 p q \sigma^{2}}{\pi \lambda}\right)^{1 / 2} f\left(N_{b}\right)\right\}+\log \left(\frac{\Delta \sigma_{0}}{p}\right)-3 \varepsilon_{b} .
$$

This theoretical relationship is plotted in Fig. 8 against the observation, considering the similarity rules previously discussed. One assumption employed in this analysis is to define $\varepsilon_{b}$ in the above equation. The 1964 Alaskan earthquake with $m_{b}$ of 7.6 has been determined independently by Houston and Kanamori (1986), Koyama and Zheng (1985) and Wyss and Brune (1967). This value and the seismic moment of the Alaskan earthquake is used as a reference. Three relationships are plotted in Fig. 8; where a

* Body-wave magnitude $m_{b}$ is reported routinely by the U.S. Geological Survey and the International Seismological Centre. The magnitude is slightly different from $m_{B}$ defined and evaluated for large earthquakes by Gutenberg and Richter (1954). 


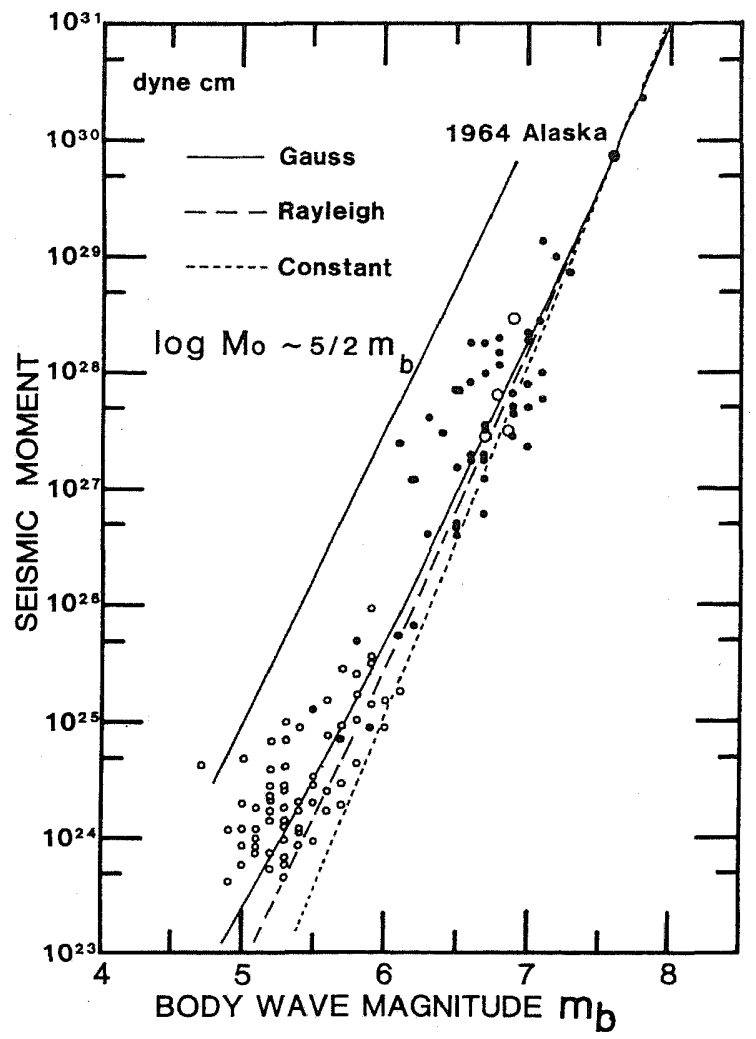

Fig. 8. Relation between body-wave magnitude $m_{b}$ and seismic moment $M_{o}$. The data (open circles) for small earthquakes are quoted from the December issue of 1990 of Earthquake Data Report by the U.S. Geological Survey. The data of large and great earthquakes along subduction zones are from Houston and Kanamori (1986) indicated by open large circles and from Koyama and Zheng (1985) by solid circles. Seismic moment is tabulated by Lay et al. (1982) and Purcaru and Berckhemer (1982). The 1964 Alaskan earthquake of $m_{b}=7.6$ is used as a reference. Theoretical $M_{o}-m_{b}$ relationships are evaluated assuming the Gauss and Rayleigh distributions for peak and trough amplitudes, and from rms amplitudes of random-phase short-waves (constant).

function $f\left(N_{b}\right)$ from the statistical theory of extremes in Eq. (5.36) is assumed to be a constant of 3 , and $f\left(N_{b}\right)$ is evaluated similarly from Eqs. (5.20) and (5.21). The value of $N_{b}$ in all three cases is similar to Eq. (5.22):

$$
N_{b}=2 \pi L / \bar{v},
$$

considering a characteristic frequency of $2 \pi$ and a corner frequency of $2 \bar{v} / L$.

It is found that the theoretical $\log M_{o}-m_{b}$ relationship in Eq. (5.36) for the Rayleigh and the Gaussian distributions explains the observed data of $M_{o}-m_{b}$ from small 
earthquakes to the greatest earthquakes in Fig. 8. On the other hand, the constant case of $f\left(N_{b}\right)=3$ systematically deviates from the observations. This is a new finding that the factor of $f\left(N_{b}\right)$ from the statistical theory of extremes is very important for the body-wave magnitude.

At first glance, the data in Fig. 8 seems to suggest a relation of $\log M_{o}=$ $(5 / 2) m_{b}+$ constant. Many studies have attempted to derive such an empirical relation by an eye-ball or a least-squares fitting. However, such an empirical relation would not have any physical significance in this case. Although the coefficient of $5 / 2$ seems to explain the observations, it would not be a consequence of the earthquake source process alone. Theoretical body-wave magnitude in Eq. (5.36) suggests that the coefficient is 3 for the $\log M_{o}-m_{b}$ relationship. This relationship is affected by the property of random-phase seismic-waves depending on the size of earthquake sources. This bias effect due to short-period seismic waves results in the deceptive coefficient of about $5 / 2$.

We have discussed the physical basis of surface-wave magnitude and body-wave magnitude based on the complex faulting process. The magnitude scale is defined from seismic-wave amplitudes in a particular frequency band, and it is evaluated from the maximum amplitude in the time domain. Therefore, the finite wavelength and the statistical theory of extremes must be considered.

\section{General Description of Complex Faulting Process}

\subsection{Similarity parameters of complex faulting process}

The physical basis of the earthquake magnitude has been understood in relation to the source parameters of the complex faulting process by the long-period and the short-period approximations. Essential parameters necessary for describing the complex faulting process are the fault length $L$, the kinematical similarity of dislocation velocity $\bar{a}$, and the stochastic similarity $\lambda \sigma^{2}$. The rest of the parameters can be estimated from the similarity rules as far as subduction zone earthquakes are concerned. Therefore, once we specify the kinematical and stochastic similarities, the earthquake source process could be scaled generally by a seismic moment $M_{o}$ (or a fault length $L$ ) in a long-period as well as in a short-period range.

We assume that $\bar{v}$ is $3 \mathrm{~km} / \mathrm{s}, \mu$ is $4 \times 10^{11} \mathrm{dyne} / \mathrm{cm}^{2}$, and that $p=q=1 / 2$ for subduction zone earthquakes, then the similarity parameters of the complex faulting process can be estimated. This is done to solve Eqs. (5.8), (5.10), (5.12), (5.31)/(5.32) and (5.33)/(5.34) simultaneously by the least-squares method.

An average dislocation velocity is thus estimated

$$
\bar{a} \simeq 30 \mathrm{~cm} / \mathrm{s} .
$$

The stochastic similarity is evaluated for the scaling P-model as

$$
\lambda \sigma^{2} \simeq 280 \mathrm{~cm}^{2} / \mathrm{s}^{3},
$$

and for the constant P-model as

$$
\sigma^{2} / \lambda \simeq 1,400 \mathrm{~cm}^{3} / \mathrm{s} .
$$


The above two values are evaluated assuming that $f(N)=2$. The constant $\epsilon$ for the definition of surface-wave magnitude is

$$
\epsilon \simeq-6.86 \text {. }
$$

An analysis indicates that $q \bar{a}$ is almost constant, so that $\bar{a}$ is dependent on the assumption of $q$. Although it seems that the stochastic similarity relates to $f(N)$, the values in Eqs. (6.2) and (6.3) are stable because the wave number of surface waves included within the wave train concerned would not drastically change among large and great earthquakes.

The difficulty in correcting for the attenuation of short-period seismic waves has caused a debate on the earthquake source spectrum used to resolve the fault heterogeneities (Boore, 1986; Houston and Kanamori, 1986; Koyama, 1985; Koyama and Izutani, 1990). The debate is focused on the effect of fault heterogeneities on the earthquake source spectrum in high frequencies. Body-wave analyses of large earthquakes support the Brune's source spectrum for large and great earthquakes (e.g., Houston and Kanamori, 1986; Kikuchi and Fukao, 1988). Brune's model (1970) has been derived intuitively from a circular fault without any fault heterogeneities. It is obvious that this model is not consistent with real earthquakes by long-and-narrow faultings (e.g., Kikuchi and Kanamori, 1986, 1991; Mori and Shimazaki, 1984; Ruff and Kanamori, 1983). The stochastic similarity in Eq. (6.2) or Eq. (6.3) suggests the importance of the small-scale fault heterogeneities on a rectangular fault plane with a geometrical similarity of $W / L \simeq 0.5$. Since the stochastic similarity thus evaluated is obtained from the empirical relations of earthquake source parameters in a relative manner, it is free from the uncertainties in whole-path propagation effects.

Although the present similarity parameters are typical values for an average earthquake of the complex faulting process, nevertheless, it would be important to constrain the deterministic part and the stochastic part of the heterogeneous earthquake source process. From the fundamental assumption of the present theory in Eqs. (2.15) and (5.17), we have a criterion for the ratio of variance dislocation velocity $\sigma^{2}$ and average dislocation velocity $\bar{a}$;

$$
\frac{4}{\lambda T_{0}}<\frac{\sigma^{2}}{\bar{a}^{2}}<\frac{\lambda T_{0}}{2},
$$

or

$$
\frac{8}{\left(\lambda T_{0}\right)^{2}}<\frac{\left\langle\Delta \sigma^{2}\right\rangle}{\Delta \sigma_{0}^{2}}<1 .
$$

When we substitute the stochastic similarity in Eq. (6.2) or Eq. (6.3) and the kinematical similarity in Eq. (6.1), the scaling P-model is valid only for large and great earthquakes with a seismic moment in the range from $1.4 \times 10^{28}$ dyne $\cdot \mathrm{cm}$ to $4.2 \times 10^{29}$ dyne $\cdot \mathrm{cm}$. Meanwhile, the constant $\mathrm{P}$-model is valid for earthquakes with a seismic moment larger than $3.3 \times 10^{26}$ dyne $\cdot \mathrm{cm}$. This suggests that the constant P-model is preferable to the scaling P-model for large and great earthquakes. This conclusion is reconfirmed in the next section by the analysis on the seismic-wave energy of large and great earthquakes. 


\subsection{Seismic-wave energy of complex faulting process}

The seismic-wave energy radiated from large and great earthquakes is another constraint for the complex faulting process. Figure 9 shows the seismic-wave energy from the recent broad-band analysis of large earthquakes plotted against the seismic moment. The relationship between S-wave energy and seismic moment from the complex faulting process is also plotted in the figure. Seismic-wave energy by Kikuchi and Fukao (1988) and by Vassiliou and Kanamori (1982) in Fig. 9 is simply doubled because their energy has been evaluated from the P-wave energy multiplying a ratio of $E_{S}^{S} / E_{S}^{P}=23.4$. The ratio is valid only for a point double-couple and not for a finitely propagating fault.

Four relationships between seismic-wave energy and seismic moment are illustrated in Fig. 9: the scaling P-model, the constant P-model assuming $\lambda=2 \pi$ and $\lambda=1$, and an empirical relation of $E_{S}=2.3 \times 10^{-5} M_{o}$. The last relation is obtained from the Brune's model. Although these relationships seem to explain the scattered data in Fig. 9, the constant P-model with $\lambda=1$ and the scaling P-model explain the observations better than the other two. It appears that the constant P-model with $\lambda=2 \pi$ generally overestimates the seismic-wave energy. Therefore, the conventional assumption that $\lambda=2 \pi$

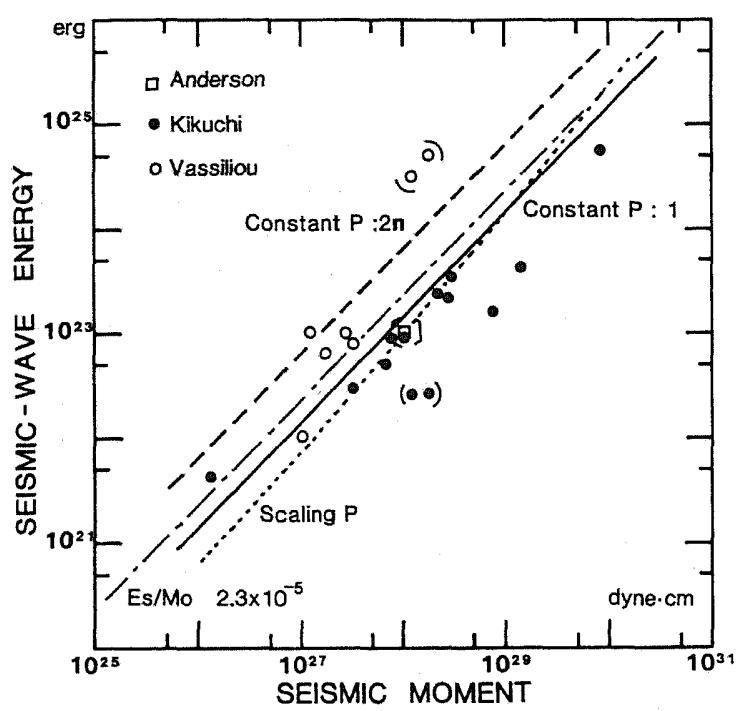

Fig. 9. Seismic-wave energy $E_{S}$ and seismic moment $M_{o}$ for large and great earthquakes in subduction zones. The data by solid circles are quoted from Kikuchi and Fukao (1988). The data by open circles are from Vassiliou and Kanamori (1982). The value of $E_{S}$ has been doubled to correspond to a finitely propagating fault. The data by a square is from strong motion records of the 1985 Michoacan earthquake in Mexico (Anderson et al., 1986). The data in brackets and square brackets are the results of the same earthquake. Theoretical relationships are indicated in the figure: the constant $P$-model $(\lambda=1)$ by a solid curve, the constant P-model $(\lambda=2 \pi)$ by a dashed curve, the scaling P-model by a dotted curve. Empirical relation by Kikuchi and Fukao (1988) is also indicated by a chained curve. 
employed in the preceding sections would not be appropriate for large earthquakes. Although we improve the assumption on the constant patch corner frequency to $\lambda=1$ instead of $\lambda=2 \pi$, all the results in the previous sections are still valid.

The above conclusion on the constant patch corner frequency can be reconfirmed, when the seismic-wave energy and surface-wave magnitude are considered. A theoretical relationship between seismic-wave energy and surface-wave magnitude can be derived from Eqs. (4.16) and (5.3) with Eq. (2.13) for small earthquakes

$$
\log E_{S} \simeq M_{S}+\log \left\{\left(1+\frac{\sigma^{2}}{\bar{a}^{2}}\right) \bar{a} \frac{\pi \mu \bar{v}^{2} p}{4 \beta^{3}} g(\chi)\right\}-\epsilon,
$$

where $g(\chi)$ is found in Eq. (3.3). Similarly, a relationship for large earthquakes can be obtained from Eqs. (4.16) and (5.5) with Eq. (2.13)

$$
\log E_{S} \simeq \frac{3}{2} M_{S}+\log \left\{\left(1+\frac{\sigma^{2}}{\bar{a}^{2}}\right) \bar{a}^{1 / 2} \frac{\mu \bar{v}}{8 \beta^{3} q^{1 / 2}} g(\chi)\right\}-\frac{3}{2} \epsilon .
$$

A theoretical relationship for great earthquakes can be derived from the constant P-model similarly from Eqs. (4.16), (5.30), and (2.13) as

$$
\begin{aligned}
\log E_{S} \simeq & 3 M_{S}+\log \left(\bar{a}^{2}+\sigma^{2}\right)-\frac{3}{2} \log \left(\frac{\sigma^{2}}{\lambda}\right) \\
& +\log \left\{\frac{\mu \omega_{s}^{3}}{128 \beta^{3} \bar{v}^{-2}} \frac{g(\chi)}{p} \frac{\pi^{3 / 2}}{(2 p q)^{1 / 2}} f^{-3}(N)\right\}-3 \epsilon,
\end{aligned}
$$

and for the scaling P-model from Eqs. (4.16), (5.29), and (2.13) as

$$
\begin{aligned}
\log E_{S} \simeq & 3 M_{S}+\log \left(\bar{a}^{2}+\sigma^{2}\right)-\frac{3}{2} \log \left(\lambda \sigma^{2}\right) \\
& +\log \left\{\frac{\mu \omega_{s}^{6}}{64 \beta^{3} \bar{v}^{2}} \frac{g(\chi)}{p} \frac{\pi^{3 / 2}}{(p q)^{1 / 2}} f^{-3}(N)\right\}-3 \epsilon .
\end{aligned}
$$

Figure 10 shows the relationships between seismic-wave energy and surface-wave magnitude in Eqs. (6.8), (6.9), and (6.10), considering the similarity rules and seismic moment. The symbols are the same as those in Fig. 9: the solid curve indicates a relationship by the constant P-model with $\lambda=1$, the dashed curve by the constant P-model with $\lambda=2 \pi$, the dotted curve by the scaling P-model, and the chained curve by the Gutenberg and Richter's empirical relation in Eq. (4.1). The relationship in Eq. (6.8) is also illustrated by a solid curve for $M_{S}$ smaller than 7.5.

Even though we consider the untractable seismic-wave energies of large earthquakes, the scatter of the data is considerably large. Among the theoretical relationships, the one by the constant $\mathrm{P}$-model with $\lambda=1$ satisfies the general trend of the data distribution better than the others. This is further evidence which supports the constant patch corner frequency of the complex faulting process for great earthquakes. Although the assumption of $\lambda=1$ is crucial for the complex faulting process, the constant patch corner frequency model is the only one which generally satisfies the constraint from the 


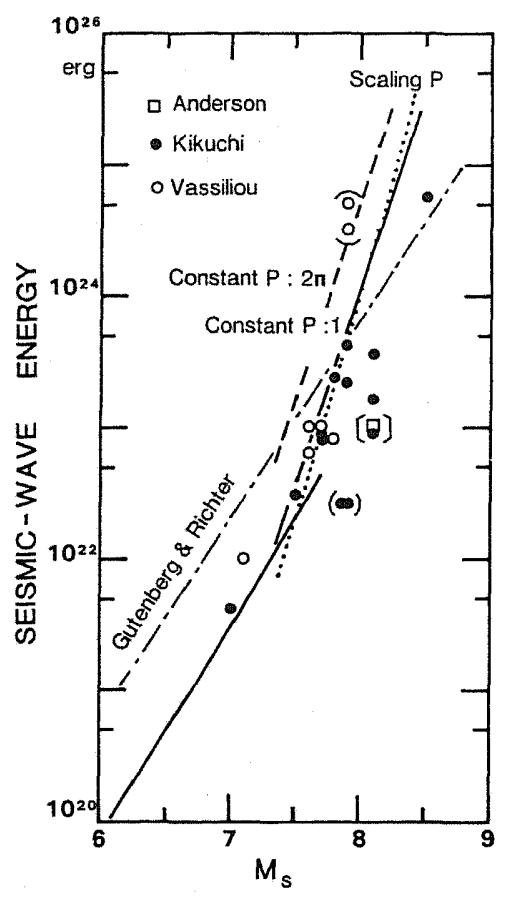

Fig. 10. Seismic-wave energy $E_{S}$ and surface-wave magnitude $M_{S}$ for large and great earthquakes in subduction zones. The data and symbols are the same as those in Fig. 9. In addition, a theoretical relationship for large earthquakes is also indicated by a solid curve in the magnitude range smaller than 7.5. Gutenberg and Richter's empirical relation is indicated by a chained curve.

seismological observations concerning the independent empirical relations between $M_{o}-M_{S}, M_{o}-S, M_{o}-m_{b}, E_{S}-M_{o}$, and $E_{S}-M_{S}$.

\subsection{Generalized scaling of earthquake source spectra}

The discussions have been developed to specify seismic moment, seismic-wave energy, and surface-wave magnitude based on the complex faulting process. The parameters necessary to describe the average source spectrum of real earthquakes have been obtained from the empirical relations, being independent of the whole-path propagation effects.

Houston and Kanamori (1986) investigated the excitation of high-frequency seismic waves of great earthquakes, and concluded that the $\omega$-square Brune model is preferable to explain the observed source spectra. Kikuchi and Fukao (1988) investigated long-period body waves by their inversion method in favor of the Brune model for source spectra of large earthquakes. Since the Brune model is intuitively considered by applying circular faults, it is not appropriate for large earthquakes which are evidently narrow faultings. This has been puzzling us for a long time.

Figure 11 shows the source spectra of great earthquakes which occurred along 


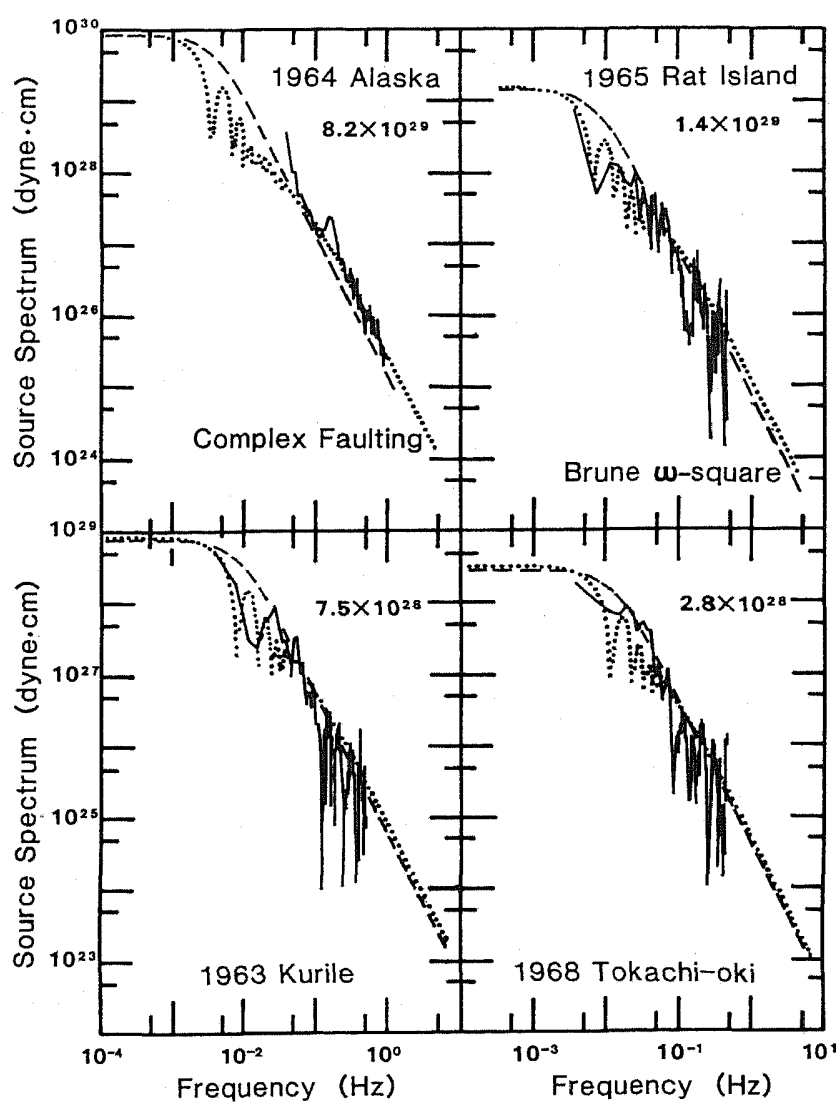

Fig. 11. Displacement source spectra of great earthquakes in subduction zones. The 1964 Alaskan spectrum is reproduced from Houston and Kanamori (1986), the 1965 Rat Island, the 1963 Kurile, and the 1968 Tokachi-oki spectra are from Kikuchi and Fukao (1988). Dotted curves illustrate the source spectrum of the complex faulting process for each earthquake assuming the seismic moment in the figure, where the constant patch corner frequency $\lambda=1(0.16 \mathrm{~Hz})$ is assumed. Dashed curves indicate the Brune's spectra.

subduction zones in the world, which have been reproduced from Houston and Kanamori (1986) and Kikuchi and Fukao (1988). Wiggling solid curves in the figures represent retrieved source spectra of great earthquakes. Dashed curves indicate Brune's source spectra appropriate for the given value of the seismic moment, where $\Delta \sigma_{0}=30$ bar and $\beta=4 \mathrm{~km} / \mathrm{s}$ are assumed. Spectra illustrated by dotted curves are evaluated from the complex faulting process applying the stochastic similarity in Eq. (6.3) with the constant patch corner frequency $\lambda=1$, which is about $0.16 \mathrm{~Hz}$.

Surprisingly, but not unreasonably, the source spectrum of the complex faulting process explains the observed spectra of great earthquakes in a high frequency range down to about $0.5-1 \mathrm{~Hz}$ in Fig. 11. The complex faulting process has been derived from 
a rectangular faulting with a geometrical similarity of $p=W / L=0.5$, therefore, it is not necessary to assume the unrealistic faulting mode on a circular fault as with the Brune's model in order to account for the source spectrum and the seismic-wave energy of large and great earthquakes. The source spectra in Fig. 11 have not been considered to constrain the parameters of the complex faulting process. Therefore, this result is further evidence which demonstrates the applicability of the present theory of the complex faulting process to real earthquake sources.

The complex faulting process characterizes the displacement source spectrum as a constant spectrum in very low-frequency, and as a spectral envelope which decays in inverse proportion to $\omega$-square after the corner frequency and which decays less rapidly in and around the patch corner frequency. This kind of source spectra has been studied by Aki (1972), Boore (1986), Gusev (1983), Koyama et al. (1982), Papageorgiou and Aki (1985), and Suzuki and Hirasawa (1984) for large earthquakes. However, these studies mainly analyzed empirical relations of seismic moment and different magnitude scales to constrain relatively the spectral content. The present theory of the complex faulting process has been constrained by the observations and the theory generally describes the amount of the spectral enhancement in high frequencies as well as the seismic-wave energy of large and great earthquakes.

Generally speaking, the complex faulting process specified by the constant patch corner frequency of $\lambda=1$ would not be valid for earthquakes with surface-wave magnitude $M_{s}$ smaller than 7. This is because the assumed patch corner frequency and the corner frequency of moderate-size earthquakes merge, and that the criterion in Eq. (6.5) is no longer valid for these earthquakes. Therefore, the present theory of the complex faulting process could only be applied to subduction zone earthquakes with magnitude larger than 7.5.

\section{Discussion and Conclusions}

The assumption of similarity has been introduced to constrain earthquake source parameters (Aki, 1967). The long-period seismology in 1970's revealed the constant stress drop for inter-plate and intra-plate earthquakes (Kanamori and Anderson, 1975), indicating different stress drops in different tectonic environments. We have studied such similarity as a general property of the complex faulting process through the longperiod approximation. The geometrical similarity, kinematical similarity and dynamical similarity are shown in relation to the well-known empirical relations in seismology.

In addition to this, the complexity of the faulting process has been demonstrated by the inversion method of long-period body waves (e.g., Baker and Langston, 1982; Kikuchi and Kanamori, 1991; Nábělek, 1985; Takeo, 1987) and of strong ground motion array-data (e.g., Fukuyama and Irikura, 1986; Hartzell and Heaton, 1986; Olson and Apsel, 1982). Generally speaking, the body-wave inversion describes earthquake faulting as a sequence of subevents by a space-time history of seismic moment release. A characteristic size of subevents would be more or less several kilometers to several tens of kilometers. Meanwhile, the strong-motion inversion has been applied for moderate-size earthquakes and resolves the distribution of seismic moment release on a fault plane as precise as several hundred meters. These two analyses are not directly 
comparable to each other because of the difference in source sizes and in wavelengths. To prescribe the complexity of earthquake source processes, we have introduced the stochastic similarity in patch corner frequency and variance dislocation velocity of the stochastic part of the complex faulting process.

The stochastic similarity of the complex faulting process is a bridge over the long-period and short-period seismology. We could determine the stochastic similarity of only subduction-zone earthquakes in this study. Even with the limited knowledge of the complexity of earthquake sources, we may demonstrate remarkable differences in aftershock distributions, $b$-values of aftershocks, strong-motion excitations, and in isoseismals from one earthquake to another. This indicates that the stochastic similarity in this study is not universal and that it would vary from place to place depending upon the tectonic environment.

The stochastic similarity in this study parametrizes the physical property of small-scale fault heterogeneities rupturing in a random manner. A fault patch specified by a larger stress drop corresponds to an asperity (Ruff and Kanamori, 1983), and that by a smaller stress drop to a barrier (Das and Aki, 1977). An important question lies on the scaling law for the fault heterogeneities whatever they are called fault patches, asperities, and/or barriers. The scaling law should answer the question on the size-number distribution of random fault patches with respect to the whole size of earthquake sources. We have concluded that the constant patch corner frequency is the best candidate to generally describe the complex faulting process of great earthquakes. However, this conclusion states that the average (statistical mean) of the size-number distribution of random fault patches is constant with respect to earthquake source sizes. This would not tell anything about the distribution itself nor the property beyond the frequency band we have defined.

A hypothesis to account for the size-number distribution of earthquakes is a fractal view of the earthquake. A power-law size distribution of earthquakes has been known as the Gutenberg and Richter formula, characterized by the $b$-value. Heterogeneities of stresses and material properties play an important role in faulting mechanics and fault geometry. Andrews (1981) and Fukao and Furumoto (1985) considered a self-similar distribution of fault heterogeneities to account for the complicated earthquake source process.

Recent studies on the complex system revealed the dynamical process of random activations specified by a self-similarity and a hierarchy structure of random clusters (Hara et al., 1992; Koyama and Hara, 1992). The theory has been applied in this study to represent the hierarchy of random fault patches. A source-oriented cutoff frequency $f_{\max }$ of acceleration spectra has been presented by the theory of random activation of fault patches. Other hypotheses to account for the cutoff frequency suggest a constant $f_{\max }$ irrespective of the size of earthquake sources. To discriminate the source-oriented $f_{\max }$ from the other hypotheses of $f_{\max }$, the scaling law of $f_{\max }$ must be studied more precisely for large and great earthquakes.

The investigation into the strong motion data would also establish the importance of the short-period seismic directivity effect on the strong ground motion of large earthquakes, and not only for $f_{\max }$. Although there has been little considered, the short-period seismic directivity effect is fundamental and essential to improve the

Vol. 42, No. 2, 1994 
precision of strong motion prediction for future large earthquakes.

Finally, we consider the energy budget of large earthquakes to conclude this study. Since the static and kinetic energies of the complex faulting process demonstrate the importance of the heterogeneous earthquake source, the discussion is not as simple as the previous studies. The static estimate of seismic energy in Eq. (4.12) can formally be rewritten as

$$
W_{h}=\frac{\Delta \sigma_{0}}{2 \mu} M_{\circ}\left\{1+\left(\frac{2}{\lambda T_{0}}\right)^{1 / 2} \frac{\sigma^{2}}{\bar{a}^{2}}\right\} .
$$

The seismic-wave energy in Eq. (4.17) is approximately expressed as

$$
E_{S} \simeq \frac{\Delta \sigma_{0}}{2 \mu} M_{o}\left\{1+\frac{\sigma^{2}}{\bar{a}^{2}}\right\} \frac{p}{4 q} \chi^{3} g(\chi),
$$

where $g(\chi)$ is a function of the velocity ratio $\chi=\bar{v} / \beta$ defined in Eq. (3.3).

Thus, we obtain a relation for the seismic-wave energy and moment ratio:

$$
\frac{E_{S}}{M_{o}}=\frac{\Delta \sigma_{0}}{2 \mu} \times 0.229\left[1+\frac{\sigma^{2}}{\bar{a}^{2}}\right],
$$

where $\chi=0.8$, and $p=q=1 / 2$ are assumed. Once we substitute the stochastic similarity and the average dislocation velocity into Eq. (7.3), the above relation is consistent with Kikuchi and Fukao's (1988) empirical relation derived from the Brune's model. We should note that the seismic-wave energy of the stochastic part is about 1.5 times larger than the deterministic part of the complex faulting process, when the constant patch corner frequency of $\lambda=1$ is considered.

The ratio of $E_{S} / W_{h}$ is equivalent to the seismic efficiency $\eta_{h}$ in case of $100 \%$ stress drop. It can be evaluated from Eqs. (7.1) and (7.2), about 0.47 for a great earthquake with $M_{S}=8.5$ and about 0.32 for $M_{S}=7.5$. This kind of variation of seismic efficiencies has been understood as that the frictional resistance against the faulting and the cohesion in the rupture front are not negligible since the ratio is smaller than unity, and that more energy is converted to seismic waves in the case of large earthquakes. This is because the energy loss due to the cohesion is restricted in the rupture front and increases in proportion to the linear length of the fault, whereas the seismic-wave energy increases in proportion to the area size of the fault. Therefore, the seismic efficiency is considered to be large for great earthquakes compared to moderate-size earthquakes.

In contrast to this understanding, the complex faulting process gives another interpletation. The contribution of fault heterogeneities on the total work $W_{h}$ is small in the case of larger earthquakes than that of smaller events: Theoretically, this can be found in the second term in braces of Eq. (7.1), where the parameter $T_{0}$ increases with respect to the size of the earthquake, reducing the contribution of the fault heterogeneities. Consequently, the energy ratio of $E_{S} / W_{h}$ increases in the case of the complex faulting process of large earthquakes. The variation in $E_{S} / W_{h}$ is essential to the deterministic and stochastic faulting processes and is not attributed merely to the frictional and cohesive conditions on the fault. 


\section{Summary}

The deterministic part and the stochastic part of the complex faulting process have been investigated theoretically and constrained empirically. The complex faulting process characterizes the earthquake source parameters by the geometrical, kinematical, and dynamical similarities for the deterministic part and by the stochastic similarity for the stochastic part. The deterministic part of the complex faulting process can be derived by a long-period or low-frequency approximation, which represents the coherent earthquake source process. The stochastic part can be derived by a short-period or high-frequency approximation, representing the small-scale incoherent earthquake source process. Therefore, we observe different properties of the heterogeneous faulting depending on frequencies and on wavelengths of seismic waves.

Displacement source spectra, acceleration spectra, and the total power of the acceleration have been formulated by this new theory of the complex faulting process. Discussion has been extended in considering the source-oriented cutoff frequency $f_{\max }$ of strong ground motion.

The total work done by the complex faulting process, the seismic energy available for wave-fields and the kinetic seismic-wave energy have been formulated considering the macroscopic source parameters as well as the stochastic source parameters. The importance of fault heterogeneities is stressed both for the seismic energy and the seismic-wave energy in this study. A discussion has also been developed on the seismic efficiency of large earthquakes in relation to the fault heterogeneities. It is shown that the seismic efficiency is not only the function of the dynamical condition on the fault but also the function of the fault heterogeneities.

The long-wavelength and the short-wavelength approximations of the complex faulting process yield the representations for surface-wave magnitude of great earthquakes, large and small earthquakes. We believe that these representations would establish the physical basis of the magnitude scale which has been empirically introduced to measure the strength of earthquakes.

The similarity rules of the complex faulting process of large earthquakes have been constrained by the empirical relations in seismology. The parameters are reconfirmed against the relation between seismic-wave energy and seismic moment of subduction zone earthquakes. The present theory of the complex faulting process. describes the general properties of large and great earthquakes along subduction zones in the world.

The author would like to acknowledge Dr. Minoru Takeo, the University of Tokyo, and Prof. Ryosuke Inoue, the Ibaraki University who recommended that he summarizes the complex faulting process of large earthquakes. Prof. Masayuki Kikuchi, Yokohama City University, inspired him and kindly sent him the original figure used in Fig. 11. The author also thanks his late uncle, Shigesaburo Kosugi and his cousin, Toshiki Kosugi for their everlasting encouragement since he was in graduate school. The memory of them is engraved on his mind forever. He would like to dedicate this study to them as a token of partakers of the sufferings, the comfort and salvation. 


\section{REFERENCES}

Abe, K., Reliable estimation of the seismic moment of large earthquakes, J. Phys. Earth, 23, 381-390, 1975 .

Abe, K., Magnitudes of large shallow earthquakes from 1904 to 1980, Phys. Earth Planet. Inter., 27, 72-92, 1981.

Adams, R. D., Survey of practice in determining magnitudes of near earthquakes, Part 2: Europe, Asia, Africa, Australia, the Pacific. World Data Center A, Report SE-8, 1-65, 1977.

Aki, K., Scaling law of seismic spectrum, J. Geophys. Res., 72, 1217-1231, 1967.

Aki, K., Scaling law of earthquake source time-function, Geophys. J. R. Astron. Soc., 31, 3-25, 1972.

Aki, K., Lecture note on strong ground motion, Res. Note Natl. Res. Cent. Disas. Prev., 80, 5-32, 1989.

Aki, K. and P. G. Richards, Quantitative Seismology Theory and Methods, Freeman \& Company, San Francisco, 932 pp., 1980.

Anderson, J. G., P. Bodin, J. N. Brune, J. Prince, S.K. Singh, R. Quaas, and M. Onate, Strong ground motion from the Mechoacan, Mexico, earthquake, Science, 233, 1043-1049, 1986.

Andrews, D. J., A stochastic fault model, 2. Time-dependent case, J. Geophys. Res., 86, 10821-10834, 1981.

Baker, J. S. and C. A. Langston, Moment tensor inversion of complex earthquakes, Geophys. $J$. R. Astron. Soc., 68, 777-803, 1982.

Bäth, M., Earthquake magnitude-recent research and current trend, Earth Sci. Rev., 17, 3115-3398, 1981.

Bendat, J. S., Principles and Application of Random Noise Theory, Wiley \& Sons, New York, 215 pp., 1958.

Boatwright, J., The seismic radiation from composite models of faulting, Bull. Seismol. Soc. Am., 78, 489-508, 1988.

Boatwright, J. and G. L. Choy, Teleseismic estimates of the energy radiated by shallow earthquakes, J. Geophys. Res., 91, 2095-2112, 1986.

Boore, D. M., Stochastic simulation of high-frequency ground motions based on seismological models of the radiated spectra, Bull. Seismol. Soc. Am., 73, 1865-1894, 1983.

Boore, D. M., Short-period P- and S-wave radiation from large earthquakes: implications for spectral scaling relations, Bull. Seismol. Soc. Am., 76, 43-64, 1986.

Boore, D. M. and W. B. Joyner, The influence of rupture incoherence on seismic directivity, Bull. Seismol. Soc. Am., 68, 283-300, 1978.

Brune, J. N., Tectonic stress and the spectra of seismic shear waves from earthquakes, J. Geophys. Res., 75, 4997-5009, 1970.

Chapman, C. H., Ray theory and its extensions: WKBJ and Maslov seismograms, Geophys. J. R. Astron. Soc., 54, 481-518, 1985.

Das, S. and K. Aki, Fault planes with barriers: a versatile earthquake model, J. Geophys. Res., 82, 5648-5670, 1977.

Dziewonski, A. M., T. A. Chou, and J. H. Woodhouse, Determination of earthquake source parameters from waveform data for studies of global and regional seismicity, J. Phys. Res., 86, 2825-2852, 1981.

Ekström, G. and A. M. Dziewonski, Evidence of bias in estimations of earthquake size, Nature, 332, 319-323, 1988. 
Fukao, Y. and M. Furumoto, Hierarchy in earthquake size distribution, Phys. Earth Planet. Inter., 37, 149-168, 1985.

Fukuyama, E. and K. Irikura, Rupture process of the 1983 Japan sea (Akita-oki) earthquake using a waveform inversion method, Bull. Seismol. Soc. Am., 76, 1623-1640, 1986.

Geller, R. G., Scaling relations for earthquake source parameters and magnitudes, Bull. Seismol. Soc. Am., 66, 1501-1523, 1976.

Geller, R. G. and H. Kanamori, Magnitude of great shallow earthquakes from 1904 to 1952, Bull. Seismol. Soc. Am., 67, 587-598, 1977.

Gusev, A. A., Descriptive statistical model of earthquake source radiation and its application to an estimation of short period strong motion, Geophys. J. R. Astron. Soc., 74, 787-808, 1983.

Gusev, A. A., Intermagnitude relationships and asperity statistics, PAGEOPH, 136, 515-527, 1991.

Gutenberg, B., Amplitudes of surface waves and magnitudes of shallow earthquakes, Bull. Seismol. Soc. Am., 35, 3-12, 1945.

Gutenberg, B. and C. F. Richter, Seismicity of the Earth, Princeton University Press, Princeton, 310 pp., 1954.

Hanks, T. C., $f_{\max }$, Bull. Seismol. Soc. Am., 72, 1867-1879, 1982.

Hanks, T. C. and R. K. McGuire, The character of high frequency strong ground motion, Bull. Seismol. Soc. Am., 71, 2071-2095, 1981.

Hara, H., O. H. Chung, and J. Koyama, Dynamical activation processes described by generalized random walks, Phys. Rev. B, 46, 838-845, 1992.

Hartzell, S. and T. Heaton, Rupture history of the 1984 Morgan Hill, California earthquake from the inversion of strong motion records, Bull. Seismol. Soc. Am., 76, 649-674, 1986.

Haskell, N.A., Total energy and energy spectral density of elastic wave radiation from propagating faults, Bull. Seismol. Soc. Am., 54, 1811-1841, 1964.

Hirasawa, T. and W. Stauder, S.J., On the seismic body waves from a finite moving source, Bull. Seismol. Soc. Am., 55, 237-262, 1965.

Honda, H., Earthquake mechanism and seismic waves, J. Phys. Earth, 10, 1-97, 1962.

Houston, H. and H. Kanamori, Source spectra of great earthquakes: teleseismic constraints on rupture process and strong motion, Bull. Seismol. Soc. Am., 76, 19-42, 1986.

Imai, I., Applied Theory of Hyperfunctions I, Science Publication, Tokyo, 210 pp., 1981.

Izutani, Y., Source parameters relevant to heterogeneity of a fault plane, J. Phys. Earth, 32, 511-529, 1984.

Kanamori, H., The energy release in great earthquakes, J. Geophys. Res., 82, 2981-2987, 1977.

Kanamori, H. and D. L. Anderson, Theoretical basis of some empirical relations in seismology, Bull. Seismol. Soc. Am., 65, 1073-1095, 1975.

Kawase, H. and K. Aki, Topography effect at the critical SV-wave incidence: possible explanation of damage pattern by the Whittier Narrows, California earthquake of 1 October 1987, Bull. Seismol. Soc. Am., 80, 1-22, 1990.

Kikuchi, M. and Y. Fukao, Seismic wave energy inferred from long-period body wave inversion, Bull. Seismol. Soc. Am., 78, 1707-1724, 1988.

Kikuchi, M. and H. Kanamori, Inversion of complex body waves-II, Phys. Earth Planet. Inter., 43, 205-222, 1986.

Kikuchi, M. and H. Kanamori, Inversion of complex body waves-III, Bull. Seismol. Soc. Am., 81, 2335-2350, 1991.

Koyama, J., Earthquake source time function from coherent and incoherent rupture, Tectonophysics, 118, 227-242, 1985.

Vol. 42, No. 2, 1994 
Koyama, J., Time-dependent moment tensor inversion for the 1983 Japan sea earthquake, Zisin Ser. 2, 40, 405-416, 1987.

Koyama, J. and H. Hara, Scaled Langevin equation to describe $1 / f^{\alpha}$ spectrum, Phys. Rev. A, 46, 1844-1849, 1992.

Koyama, J. and Y. Izutani, Seismic excitation and directivity of short-period body waves from a stochastic fault model, Tectonophysics, 175, 67-79, 1990.

Koyama, J. and N. Shimada, Physical basis of earthquake magnitudes: an extreme value of seismic amplitudes from incoherent fracture of random fault patches, Phys. Earth Planet. Inter., 40, 301-308, 1985.

Koyama, J. and S. H. Zheng, Excitation of short period body waves by great earthquakes, Phys. Earth Planet. Inter., 37, 108-123, 1985.

Koyama, J. and S.H. Zheng, Seismic intensity distribution of shallow earthquakes due to rupture velocities and faulting modes, Acta Seismol. Sinica, 13, 190-201, 1991.

Koyama, J., M. Takemura, and Z. Suzuki, A scaling model for quantification of earthquakes in and near Japan, Tectonophysics, 84, 3-16, 1982.

Lay, T., H. Kanamori, and L. Ruff, The asperity model and the nature of large subduction zone earthquakes, Earthq. Predict. Res., 1, 3-71, 1982.

Lee, W. H. K. and R. J. Wetmiller, Survey of practice in determining magnitudes of near earthquakes, Part 1: North, Central, and South America. World Data Center A, Report SE-9, 1-101, 1978.

Lighthill, M.J., Introduction to Fourier Analysis and Generalized Functions, Cambridge University Press, London, 45 pp., 1959.

Maruyama, T., On the force equivalents of dynamic elastic dislocations with reference to the earthquake mechanism, Bull. Earthq. Res. Inst., Univ. Tokyo, 41, 467-486, 1963.

Mikumo, T. and T. Miyatake, Numerical modeling of realistic fault rupture processes, in Strong Motion Synthetics, ed. B.A. Bolt, pp. 91-151, Academic Press, London, 1987.

Mori, J. and K. Shimazaki, High stress drops of short-period subevents from the 1968 Tckachi-oki earthquake as observed on strong-motion records, Bull. Seismol. Soc. Am., 74, 1529-1544, 1984.

Nábělek, J., Geometry and mechanism of faulting of the 1980 El Asnam, Algeria, earthquake from inversion of teleseismic body waves and comparison with field observations, J. Geophys. Res., 90, 12713-12728, 1985.

Noguchi, S. and K. Abe, Earthquake source mechanism and $M_{s}-m_{b}$ relation, Zisin, Ser. 2, 30, 487-507, 1977.

Ohnaka, M., Y. Kuwahara, and K. Yamamoto, Constitutive relations between dynamic physical parameters near a tip of the propagating slip zone during stick-slip shear failure, Tectonophysics, 144, 109-125, 1987.

Olson, A. and R. Apsel, Finite faults and inverse theory with applications to the 1979 Imperial Valley earthquake, Bull. Seismol. Soc. Am., 72, 1969-2001, 1982.

Orowan, E., Mechanism of seismic faulting, Geol. Soc. Am. Mem., 79, 323-345, 1960.

Papageorgiou, A. S. and K. Aki, A specific barrier model for quantitative description of inhomogeneous faulting and the prediction of strong ground motion. Part I. Description of the model, Bull. Seismol. Soc. Am., 73, 693-722, 1983.

Papageorgiou, A. S. and K. Aki, Scaling law of far-field spectra based on observed parameters of the specific barrier model, PAGEOPH, 123, 353-374, 1985.

Purcaru, G. and H. Berckhemer, A magnitude scale for very large earthquakes, Tectonophysics, 49, 189-198, 1978. 
Randoll, M.J. and L. Knopoff, The mechanism at the focus of deep earthquakes, J. Geophys. Res., 75, 4965-4976, 1970.

Rice, J. R., The mechanics of earthquake rupture, in Proc. Intern. School Phys., "Enrico Fermi", Italian Phys. Soc., LXXVIII, ed. E. Boschi and A. M. Dziewonski, pp. 555-649, North-Holland Publication, Amsterdam, 1980.

Rudnicki, J. W. and L. B. Freund, On energy radiation from seismic sources, Bull. Seismol. Soc. Am., 71, 583-595, 1981.

Ruff, L. and H. Kanamori, The rupture process and asperity distribution of three great earthquakes from long-period diffracted P-waves, Phys. Earth Planet. Inter., 31, 202-230, 1983.

Sato, H., Unified approach to amplitude attenuation and coda excitation in the randomly inhomogeneous lithosphere, PAGEOPH, 132, 1-29, 1990.

Sato, T. and T. Hirasawa, Body wave spectra from propagating shear cracks, J. Phys. Earth, 21, 415-431, 1973.

Savage, J. C., Relation of corner frequency to fault dimensions, J. Geophys. Res., 77, 3788-3795, 1972.

Savage, J. C. and M. D. Wood, The relation between apparent stress and stress drop, Bull. Seismol. Soc. Am., 61, 1381-1388, 1971.

Scholz, C. H., The Mechanics of Earthquakes and Faulting, Cambridge University Press, New York, 221 pp., 1990.

Sipkin, S. A., Estimation of earthquake source parameters by the inversion of waveform data: synthetic waveforms, Phys. Earth Planet. Inter., 30, 242-259, 1982.

Spudich, P. and L. N. Frazer, Use of ray theory to calculate high-frequency radiation from earthquake sources having spatially variable rupture velocity and stress drop, Bull. Seismol. Soc. Am., 74, 2061-2082, 1984.

Suzuki, Y. and T. Hirasawa, Strong motions from a stochastic model of faulting, Proc. 8th World Conference Earthquake Engineering, San Francisco, Vol. II, 481-488, 1984.

Takemura, M. and J. Koyama, A scaling model for low-frequency earthquakes-relation of source spectra between tsunami earthquakes and small low-frequency earthquakes, Zisin, Ser. 2, 36, 323-333, 1983.

Takeo, M., An inversion method to analyze the rupture processes of earthquakes using near-field seismograms, Bull. Seismol. Soc. Am., 77, 490-513, 1987.

Toro, G. R. and R. K. McGuire, An investigation into earthquake ground motion characteristics in eastern north America, Bull. Seismol. Soc. Am., 77, 468-489, 1987.

Tse, S. T. and J. R. Rice, Crustal earthquake instability in relation to the depth variation of frictional slip properties, J. Geophys. Res., 91, 9452-9472, 1986.

Utsu, T., Catalog of large earthquakes in the region of Japan from 1885 through 1980, Bull. Earthq. Res. Inst., Univ. Tokyo, 57, 401-463, 1982a.

Utsu, T., Relationships between earthquake magnitude scales, Bull. Earthq. Res. Inst., Univ. Tokyo, 57, 465-497, 1982b.

Utsu, T. and A. Seki, A relation between the area of aftershock region and the energy of main shock, Zisin, Ser. 2, 7, 233-240, 1954.

Vassiliou, M. S. and H. Kanamori, The energy release in earthquakes, Bult. Seismol. Soc. Am., 72, 371-387, 1982.

Wyss, M. and J. N. Brune, The Alaska earthquake of 28 March 1964: a complex multiple rupture, Bull. Seismol. Soc. Am., 57, 1017-1023, 1967. 


\section{APPENDIX A}

\section{Static Estimate of Seismic Energy}

Suppose that the stress change is given by $\sigma_{i j}(\vec{\xi})$ and that the displacement associated with the stress change is represented by $u_{i}(\vec{\xi}, t)$ at a point $\vec{\xi}$ and a time $t$. The total work is defined as (Rudnicki and Freund, 1981)

$$
W_{0}=-\int_{-\infty}^{\infty} \mathrm{d} t \int_{S} \mathrm{~d} S \sigma_{i j} \gamma_{j} \dot{u}_{i}
$$

where the dot stands for time derivative, $S$ is the source area, and $\gamma_{j}$ is the unit normal to $S$.

If an earthquake source is considered as a shear faulting, the displacement can be assumed only in the $x_{1}$ direction, $u_{i}(\vec{\xi}, t)=u(\vec{\xi}, t) \delta_{i 1}$, where $\delta_{i 1}$ is Kronecker's delta and the stress change is only on the $x_{1} x_{2}$-plane. When we introduce a local stress drop $\Delta \sigma(\vec{\xi})$ taking an opposite sign to $\sigma_{i j}$ in Eq. (A.1), and an associated dislocation $D(\xi, t)$ on a heterogeneous fault plane, then we have

$$
\begin{aligned}
& \Delta \sigma(\vec{\xi})=\Delta \sigma_{0}+\delta \sigma(\vec{\xi}), \\
& \dot{D}(\vec{\xi}, t)=\bar{a}+\delta \dot{D}(\vec{\xi}, t),
\end{aligned}
$$

where $\Delta \sigma_{0}$ is an average stress drop and $\bar{a}$ is an average dislocation velocity on the fault. Parameters $\delta \sigma$ and $\delta \dot{D}$ represent the fluctuation of stress drop and dislocation velocity in random fault patches, which are specified by zero mean. The work done by this heterogeneous faulting is

$$
\begin{aligned}
W_{h} & =\frac{1}{2} \int_{-\infty}^{\infty} \mathrm{d} t \int_{S} \mathrm{~d} S \Delta \sigma(\vec{\xi}) \dot{D}(\vec{\xi}, t) \\
& =\frac{1}{2} \int_{-\infty}^{\infty} \mathrm{d} t \int_{S} \mathrm{~d} S\left\{\Delta \sigma_{0} \vec{a}+\delta \sigma(\vec{\xi}) \delta \dot{D}(\vec{\xi}, t)\right\} .
\end{aligned}
$$

The second term in the right hand side of the above equation is bounded by Cauchy-Schwarz's inequality, and we have

$$
\delta W \leq \frac{1}{2}\left\{\int_{S} \mathrm{~d} S \int \mathrm{d} t \delta \sigma^{2}(\vec{\xi})\right\}^{1 / 2}\left\{\int_{S} \mathrm{~d} S \int \mathrm{d} t \delta \dot{D}^{2}(\vec{\xi}, t)\right\}^{1 / 2} .
$$

From the definition of variance stress drop in Eq. (2.14) and from the definition of variance dislocation velocity in Eq. (2.5), Eq. (A.5) is rewritten

$$
\delta W \leq \frac{1}{2}\left\{\frac{\left\langle\Delta \sigma^{2}\right\rangle^{2}}{2 \mu^{2}} L^{2} W^{4} \lambda T_{0}\right\}^{1 / 2} .
$$

Considering seismic moment in terms of stress drop in Eq. (2.13), Eq. (A.6) reduces to

$$
\delta W \leq \frac{\Delta \sigma_{0}}{2 \mu} M_{0}\left\{\frac{\left\langle\Delta \sigma^{2}\right\rangle}{\Delta \sigma_{0}^{2}}\left(\frac{\lambda T_{0}}{2}\right)^{1 / 2}\right\} .
$$


APPENDIX B

Time-Domain Amplitude and Spectral Amplitude

Consider a random variable $x(t)$. If $\omega_{s}$ is a characteristic frequency, the filtered random variable $x\left(t ; \omega_{s}\right)$ is

$$
\begin{aligned}
x\left(t ; \omega_{s}\right) & =\int_{-\infty}^{\infty} x(t-\tau) \exp \left(i \omega_{s} \tau\right) \mathrm{d} \tau \\
& =\frac{1}{2 \pi} \int_{-\infty}^{\infty} X(\omega) \delta\left(\omega-\omega_{s}\right) \exp (i \omega t) \mathrm{d} \omega,
\end{aligned}
$$

where $X(\omega)$ is the Fourier transform of $x(t)$

$$
X(\omega)=\int_{-\infty}^{\infty} x(t) \exp (-i \omega t) \mathrm{d} t,
$$

and $\delta$ is the Dirac delta function. Equation (B.1) is directly derived from the convolution theorem of the Fourier transform.

Suppose that $x\left(t ; \omega_{s}\right)$ represents coherent waves with a signal duration $T$. Figure B1 illustrates such a waveform. Then the time domain amplitude $A_{s}$ of the waves provides an estimate of the spectrum as

$$
\begin{aligned}
\left|\int_{-T / 2}^{T / 2} x\left(t ; \omega_{S}\right) \exp (-i \omega t) \mathrm{d} t\right| & =\left|\int_{-T / 2}^{T / 2} A_{s} \exp \left\{i\left(\omega_{s} t+\phi\right)\right\} \exp (-i \omega t) \mathrm{d} t\right| \\
& \simeq A_{s} T,
\end{aligned}
$$

where $\phi$ is the phase.

From Eq. (B.1) the spectrum of the filtered variable is

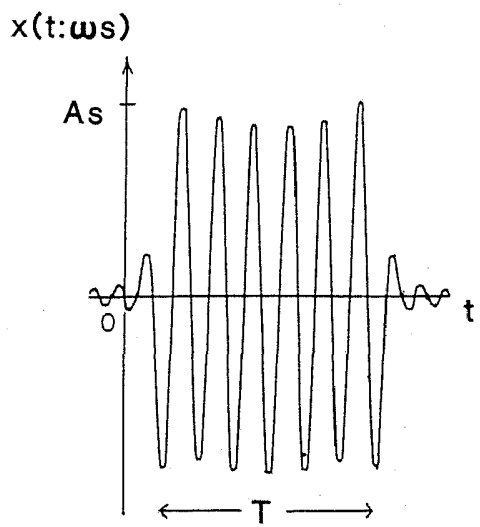

Fig. B1. An example of filtered signal $x\left(t ; \omega_{s}\right)$ with continuous phase. Signal duration is $T$ in this case.

Vol. 42, No. 2, 1994 


$$
\begin{aligned}
\int_{-T / 2}^{T / 2} x\left(t ; \omega_{s}\right) \exp (-i \omega t) \mathrm{d} t= & \int_{-T / 2}^{T / 2} \mathrm{~d} t \exp (-i \omega t) \\
& \times \frac{1}{2 \pi} \int_{-\infty}^{\infty} \mathrm{d} \omega^{\prime} X\left(\omega^{\prime}\right) \delta\left(\omega^{\prime}-\omega_{s}\right) \exp \left(i \omega^{\prime} t\right) \\
= & \frac{1}{2 \pi} \int_{-\infty}^{\infty} \mathrm{d} \omega^{\prime} X\left(\omega^{\prime}\right) \delta\left(\omega^{\prime}-\omega_{s}\right) \frac{2 \sin \left(\omega-\omega^{\prime}\right) T / 2}{\omega-\omega^{\prime}}
\end{aligned}
$$

If the signal duration $T$ is very small, the fraction in the right hand side of Eq. (B.4) tends to be $T$. Equating (B.3) and (B.4) yields a relationship of the time domain amplitude $A_{s}$ as

$$
A_{s}=\left|X\left(\omega_{s}\right)\right| / 2 \pi \text {. }
$$

When $T$ is large, the fraction in Eq. (B.4) is approximated as

$$
\begin{aligned}
\lim _{T \rightarrow \infty} \frac{\sin x T}{\pi x} & =\frac{1}{2 \pi} \int_{-\infty}^{\infty} \exp (i x t) \mathrm{d} t \\
& =\delta(x) .
\end{aligned}
$$

Therefore, the time domain amplitude is related to the spectrum as

$$
A_{s}=\left|X\left(\omega_{s}\right)\right| / T \text {. }
$$

Equation (B.7) may not be mathematically straightforward. However, a formal calculus of hyperfunctions leads us to Eq. (B.7) (Imai, 1981; Lighthill, 1959). This could be proved as follows: $\delta$ function can be defined as the extreme of a Gaussian function

$$
\delta\left(\omega-\omega_{s}\right)=\lim _{\Delta \rightarrow 0} \frac{1}{\sqrt{2 \pi \Delta^{2}}} \exp \left\{-\frac{\left(\omega-\omega_{s}\right)^{2}}{2 \Delta^{2}}\right\} .
$$

Substituting Eq. (B.8) into Eq. (B.4), we perform the integration by the steepest descent method (Koyama and Zheng, 1985). Then when we take the extreme of $\Delta \rightarrow 0$, Eq. (B.7) is obtained.

\section{APPENDIX C}

\section{$\boldsymbol{R}_{\mathrm{ms}}$ Amplitude of Random-Phase Seismic Waves}

Suppose that $x\left(t ; \omega_{s}\right)$ is characterized by a filtered signal with a random phase. Examples of such wave trains can be found in Fig. C1. Autocovariance of the filtered variable is expressed by applying Eq. (B.1) as 


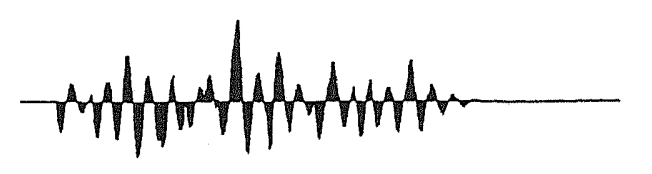

11111111111111111111111111111

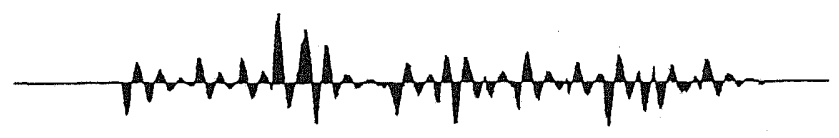

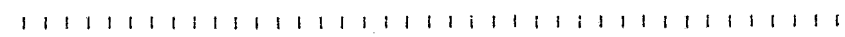

Fig. C1. Examples of filtered signal with random phase. Component wavelet is characterized by a period of two tick marks. Many wavelets are superimposed in a random manner. Upper and lower traces include the same number of wavelets, where wavelets overlap much more in the upper case.

$$
\begin{aligned}
\int_{-\infty}^{\infty} x\left(t+\tau ; \omega_{s}\right) x\left(t ; \omega_{s}\right) * \mathrm{~d} t= & \int_{-\infty}^{\infty} \mathrm{d} t\left\{\frac{1}{2 \pi} \int_{-\infty}^{\infty} X\left(\omega^{\prime}\right) \delta\left(\omega^{\prime}-\omega_{s}\right) \exp \left\{i \omega^{\prime}(t+\tau)\right\} \mathrm{d} \omega^{\prime}\right\} \\
& \times\left\{\frac{1}{2 \pi} \int_{-\infty}^{\infty} X(\omega) \delta\left(\omega-\omega_{s}\right) \exp (i \omega t) \mathrm{d} \omega\right\}^{*} \\
= & \frac{1}{2 \pi} \int_{-\infty}^{\infty} X(\omega) X(\omega)^{*} \delta\left(\omega-\omega_{s}\right) \exp (i \omega \tau) \mathrm{d} \omega
\end{aligned}
$$

where $*$ is understood as the complex conjugate. The formal calculus has been employed similarly as Appendix B to derive Eq. (C.1).

When $\tau$ tends to zero, Eq. (C.1) gives Parseval's equality for the filtered variable

$$
\begin{aligned}
\int_{-\infty}^{\infty}\left|x\left(t ; \omega_{s}\right)\right|^{2} \mathrm{~d} t & =\frac{1}{2 \pi} \int_{-\infty}^{\infty} X(\omega) X(\omega)^{*} \delta\left(\omega-\omega_{s}\right) \mathrm{d} \omega \\
& =\frac{1}{\pi}\left|X\left(\omega_{s}\right)\right|^{2} .
\end{aligned}
$$

\section{LIST OF SYMBOLS}

$\bar{a}$, average dislocation velocity;

$A_{\text {rms }}$, root-mean square amplitude;

$c, \mathrm{P}$ - or S-wave velocity;

$\bar{d}$, characteristic fault-patch size;

$E_{S}, E_{S}^{P}, E_{S}^{S}$, total seismic-, P-, S-wave energies;

$f(N)$, function from statistical theory of

extremes;
$A_{\max }$, maximum amplitude in time domain;

$B_{c}$, displacement source spectrum;

$c_{0}$, correction for source amplitude;

$D$, dislocation function;

$f_{\max }$, characteristic cutoff frequency in $\mathrm{Hz}$;

$g(\chi)$, function due to rupture velocity;

Vol. 42, No. 2, 1994 
$G_{h}, G_{f}$, stochastic, fractal acceleration spectra;

$L, W, S$, fault length, width, area size;

$M_{o}$, seismic moment;

$p$, ratio of $W / L$;

$q$, ratio of $T_{0}$ to $W / \bar{v}$;

$T_{0}$, rise time for dislocation;

$\bar{v}$, average rupture velocity;

$W_{t}$, total work done by an earthquake;

$\Delta \sigma_{0}$, average stress drop;

$\epsilon, \varepsilon$, constant for $M_{S}$ definitions;

$\zeta_{1}, \zeta_{2}$, characteristic angular frequencies of fault patches;

$\theta, \phi$, polar angle and azimuth angle;

$\lambda$, patch corner (angular) frequency;

$\sigma^{2}$, variance dislocation velocity;

$\chi$, ratio of $\bar{v} / \beta$;

$\omega_{b}$, characteristic angular frequency for body-waves;

$\omega_{m}$, angular frequency of $f_{\max } ;$ $k_{c}$, wave number for P- or S-waves;

$m_{b}$, body-wave magnitude;

$M_{S}, M_{s}$, surface-wave magnitude;

$P_{a}$, total power of acceleration;

$R_{\phi \phi}$, radiation pattern coefficient;

$u_{r}, u_{\theta}, u_{\phi}$, displacement components;

$W_{0}, W_{h}$, energy available for seismic-waves;

$\alpha, \beta, \mathrm{P}$ - and S-wave velocity;

$\left\langle\Delta \sigma^{2}\right\rangle$, variance stress drop;

$\varepsilon_{b}$, constant for $m_{b}$ definition;

$\eta_{0}, \eta_{h}$, seismic efficiency;

$\kappa$, fractal dimension of random fault patches;

$\mu, \rho$, rigidity and density;

$\sigma_{1}, \sigma_{2}, \sigma_{f}$, initial, final and frictional stresses;

$\omega$, angular frequency;

$\omega_{c}, \omega_{b}$, angular corner frequency, that for S-waves;

$\omega_{s}$, characteristic angular frequency for surface-waves. 